\title{
Landweber exact formal group laws and smooth cohomology theories
}

\author{
ULRICH BUNKE \\ THOMAS SCHICK \\ INGO SCHRÖDER \\ MORITZ WIETHAUP
}

\begin{abstract}
The main aim of this paper is the construction of a smooth (sometimes called differential) extension $\widehat{M U}$ of the cohomology theory complex cobordism $M U$, using cycles for $\widehat{M U}(M)$ which are essentially proper maps $W \rightarrow M$ with a fixed $U$-structure and $U$-connection on the (stable) normal bundle of $W \rightarrow M$.

Crucial is that this model allows the construction of a product structure and of pushdown maps for this smooth extension of $M U$, which have all the expected properties.

Moreover, we show that $\widehat{R}(M):=\widehat{M U}(M) \otimes_{M U^{*}} R$ defines a multiplicative smooth extension of $R(M):=M U(M) \otimes_{M U^{*}} R$ whenever $R$ is a Landweber exact $M U^{*}{ }_{-}$ module, by using the Landweber exact functor principle. An example for this construction is a new way to define a multiplicative smooth $K$-theory.
\end{abstract}

$55 \mathrm{~N} 20,57 \mathrm{R} 19$

\section{Introduction}

Smooth (also called differentiable) extensions of generalized cohomology theories recently became an intensively studied mathematical topic with many applications ranging from arithmetic geometry to string theory. Foundational contributions are Cheeger and Simons [7], Brylinski [2] (in the case of ordinary cohomology) and Hopkins and Singer [14]. The latter paper gives among many other results a general construction of smooth extensions in homotopy theoretic terms. For cohomology theories based on geometric or analytic cycles there are often alternative models. This applies in particular to ordinary cohomology whose smooth extension has various different realizations (see Cheeger and Simons [7], Gajer [12], Brylinski [2], Dupont and Ljungmann [8], Hopkins and Singer [14] and Bunke, Kreck and Schick [3]). The papers by Simons and Sullivan [21] or Bunke and Schick [5] show that all these realizations are isomorphic. 
An example of a cycle model of a smooth extension of a generalized cohomology theory is the model of smooth $K$-theory introduced by Bunke and Schick [4]; see also Freed [10] and Freed and Hopkins [11]. The present paper contributes geometric models of smooth extensions of cobordism theories, where the case of complex cobordism theory $M U$ is of particular importance. In [5] we obtain general results about uniqueness of smooth extensions which in particular apply to smooth $K$-theory and smooth complex cobordism theory $\widehat{M U}$. In detail, any two smooth extensions of complex cobordism theory or complex $K$-theory which admit an integration along $\int: S^{1} \times M \rightarrow M$ are isomorphic by a unique isomorphism compatible with $\int$. In case of multiplicative extensions the isomorphism is automatically multiplicative. Note that the extension $\widehat{M U}$ constructed in the present paper has an integration and is multiplicative.

We expect that our model $\widehat{M U}$ of the smooth extension of $M U$ is uniquely isomorphic to the one given by Hopkins and Singer [14]. So far this fact can not immediately be deduced from the above uniqueness result since for the latter model the functorial properties of the integration map have not been developed yet in sufficient detail. However, for the uniqueness of the even part we do not need the integration. Therefore in even degrees our extension $\widehat{M U}$ is uniquely isomorphic to the model in [14].

An advantage of geometric or analytic models is that they allow the introduction of additional structures like products, smooth orientations and integration maps with good properties. These additional properties are fundamental for applications. In [14, 4.10] methods for integrating smooth cohomology classes were discussed, but further work will be required in order to turn these ideas into constructions with good functorial properties.

In the case of smooth ordinary cohomology the product and the integration have been considered in various places (see eg Cheeger and Simons [7], Dupont and Ljungmann [8] and Brylinski [2]) (here smooth orientations are ordinary orientations). The case of smooth $K$-theory, discussed in detail in Bunke and Schick [4], shows that in particular the theory of orientations and integration is considerably more complicated for generalized cohomology theories.

In the present paper we construct a multiplicative extension of the complex cobordism cohomology theory $M U$. Furthermore, we introduce the notion of a smooth $M U-$ orientation and develop the corresponding theory of integration. The same ideas could be applied with minor modifications to other cobordism theories.

For an $M U^{*}$-module $R$ one can try to define a new cohomology theory $R^{*}(X):=$ $M U^{*}(X) \otimes_{M U^{*}} R$ for finite CW-complexes $X$. By Landweber's famous result [16] this construction works and gives a multiplicative complex oriented cohomology theory 
provided $R$ is a ring over $M U^{*}$ which is in addition Landweber exact. In Theorem 2.5 we observe that by the same idea one can define a multiplicative smooth extension $\widehat{R}(X):=\widehat{M U}(X) \otimes_{M U^{*}} R$ of $R$. It immediately follows that this smooth extension admits an integration for smoothly $M U$-oriented proper submersions.

In this way we considerably enlarge the class of examples of generalized cohomology theories which admit multiplicative extensions and integration maps. The construction can, for example, be applied to Landweber exact elliptic cohomology theories (see Landweber, Ravenel and Stong [17] and Franke [9]) and complex $K$-theory ${ }^{1}$.

In Section 2 we review the main result of Landweber [16] and the definition of a smooth extension of a generalized cohomology theory. We state the main result asserting the existence of a multiplicative smooth extension of $M U$ with orientations and integration. Then we realize the idea sketched above and construct a multiplicative smooth extension for every Landweber exact formal group law.

In Section 3 we review the standard constructions of cobordism theories using homotopy theory on the one hand, and cycles on the other. Furthermore, we review the notion of a genus.

In Section 4 we construct our model of the multiplicative smooth extension of complex cobordism. Furthermore, we introduce the notion of a smooth $M U$-orientation and construct the integration map.

Acknowledgements Thomas Schick was partially funded by the Courant Research Center "Higher order structures in Mathematics" within the German initiative of excellence. Ingo Schröder and Moritz Wiethaup were partially funded by DFG GK 535 "Groups and Geometry".

\section{The Landweber construction and smooth extensions}

\subsection{The Landweber construction}

2.1.1 Let $X \mapsto M U^{*}(X)$ denote the multiplicative cohomology theory (defined on the category of $\mathrm{CW}$-complexes) called complex cobordism. We fix an isomorphism $M U^{*}\left(\mathbb{C} \mathbb{P}^{\infty}\right) \cong M U^{*} \llbracket x \rrbracket$. The Künneth formula then gives $M U^{*}\left(\mathbb{C} \mathbb{P}^{\infty} \times \mathbb{C} \mathbb{P}^{\infty}\right) \cong$ $M U^{*} \llbracket x, y \rrbracket$.

\footnotetext{
${ }^{1}$ It is an interesting problem to understand explicitly the relation with the model [4]. Note that we abstractly know that the smooth extensions are isomorphic by [5].
} 
The tensor product of line bundles induces an $H$-space structure $\mu: \mathbb{C P} \mathbb{P}^{\infty} \times \mathbb{C P}^{\infty} \rightarrow$ $\mathbb{C} \mathbb{P}^{\infty}$. Under the above identifications the map $\mu^{*}: M U^{*} \llbracket z \rrbracket \rightarrow M U^{*} \llbracket x, y \rrbracket$ is determined by the element $f(x, y):=\mu^{*}(z) \in M U^{*} \llbracket x, y \rrbracket$.

By a result of Quillen [19] the pair $\left(M U^{*}, f\right)$ is a universal formal group law. This means that, given a commutative ring $R$ and a formal group law $g \in R \llbracket x, y \rrbracket$, there exists a unique ring homomorphism $\theta: M U^{*} \rightarrow R$ such that $\theta_{*}(f)=g$.

2.1.2 Let $R$ be a commutative ring over $M U^{*}$. Then one can ask if the functor $X \mapsto M U^{*}(X) \otimes_{M U^{*}} R$ is a cohomology theory on the category of finite CW-complexes. The result of Landweber [16] determines necessary and sufficient conditions. A ring which satisfies these conditions is called Landweber exact.

2.1.3 Actually, Landweber shows a stronger result which is crucial for the present paper. For any space or spectrum $X$ the homology $M U_{*}(X)$ has the structure of a comodule over the coalgebra $M U_{*} M U$ in $M U^{*}$-modules. By duality, if $X$ is finite, then $M U^{*}(X) \cong M U_{*}(S(X))$ also has a comodule structure, where $S(X)$ denotes the Alexander-Spanier dual (see Adams [1]) of $X$.

Theorem 2.1 (Landweber [16]) Let $M$ be a finitely presented $M U^{*}$-module which has the structure of a comodule over $M U_{*} M U$, and consider a Landweber exact formal group law $(R, g)$ so that in particular $R$ is a ring over $M U^{*}$. Then for all $i \geq 1$ we have $\operatorname{Tor}_{i}^{M U^{*}}(M, R)=0$.

\subsection{Smooth cohomology theories}

2.2.1 In the present subsection $B$ denotes a compact smooth manifold. Let $N$ be a $\mathbb{Z}$-graded vector space over $\mathbb{R}$. We consider a generalized cohomology theory $h$ with a natural transformation of cohomology theories $c: h(B) \rightarrow H(B, N)$, where $H(B, N)$ is ordinary cohomology with coefficients in $N$. The natural universal example is given by $N:=h^{*} \otimes \mathbb{R}$, where $c$ is the canonical transformation. Let $\Omega(B, N):=\Omega(B) \otimes_{\mathbb{R}} N$, where $\Omega(B)$ denotes the smooth real differential forms on $B$. Note that this definition only coincides with the corresponding definition of $N$-valued forms in [5] if $N$ is degree-wise finite-dimensional. By $d R: \Omega_{d=0}(B, N) \rightarrow H(B, N)$ we denote the de Rham map which associates to a closed form the corresponding cohomology class. To a pair $(h, c)$ we associate the notion of a smooth extension $\hat{h}$. Note that manifolds in the present paper may have boundaries.

Definition 2.2 A smooth extension of the pair $(h, c)$ is a functor $B \rightarrow \widehat{h}(B)$ from the category of compact smooth manifolds to $\mathbb{Z}$-graded groups together with natural transformations 
(1) $R: \hat{h}(B) \rightarrow \Omega_{d=0}(B, N)$ (curvature)

(2) $I: \hat{h}(B) \rightarrow h(B)$ (forget smooth data)

(3) $a: \Omega(B, N) / \operatorname{im}(d) \rightarrow \widehat{h}(B)$ (action of forms).

These transformations are required to satisfy the following axioms:

(1) The following diagram commutes:

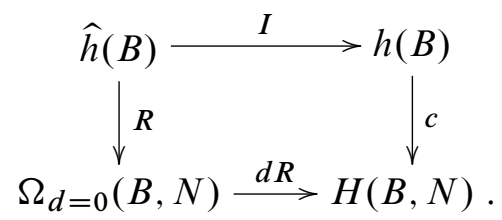

(2) $R \circ a=d$.

(3) $a$ is of degree 1 .

(4) The sequence

$$
h(B) \stackrel{c}{\rightarrow} \Omega(B, N) / \operatorname{im}(d) \stackrel{a}{\rightarrow} \hat{h}(B) \stackrel{I}{\rightarrow} h(B) \rightarrow 0 .
$$

is exact.

2.2.2 If $h$ is a multiplicative cohomology theory, then one can consider a $\mathbb{Z}$-graded ring $R$ over $\mathbb{R}$ and a multiplicative transformation $c: h(B) \rightarrow H(B, R)$. In this case we also talk about a multiplicative smooth extension $\hat{h}$ of $(h, c)$.

Definition 2.3 A smooth extension $\hat{h}$ of $(h, c)$ is called multiplicative, if $\hat{h}$ together with the transformations $R, I, a$ is a smooth extension of $(h, c)$, and in addition

(1) $\hat{h}$ is a functor to $\mathbb{Z}$-graded rings,

(2) $R$ and $I$ are multiplicative,

(3) $a(\omega) \cup x=a(\omega \wedge R(x))$ for $x \in \widehat{h}(B)$ and $\omega \in \Omega(B, R) / \operatorname{im}(d)$.

2.2.3 The first goal of the present paper is the construction of a multiplicative smooth extension of the pair $(M U, c)$, where $c: M U^{*}(B) \rightarrow M U^{*}(B) \otimes_{\mathbb{Z}} \mathbb{R} \cong H^{*}(B, M U \mathbb{R})$ is the canonical natural transformation (see Section 3.4.7). The following theorem is a special case of Theorem 4.21 which gives a construction of multiplicative smooth extensions of more general pairs $(M U, h)$.

Theorem 2.4 The pair $(M U, c)$ admits a multiplicative smooth extension.

The existence of a smooth extension also follows from [14], but there, no ring structure is constructed. 
2.2.4 In the present paper we consider smooth extensions of generalized cohomology theories defined on the category of compact manifolds. The reason lies in the fact that we want to apply the Landweber exact functor theorem. If $R$ is a generalized complex oriented cohomology theory satisfying the wedge axiom to which the Landweber exact functor theorem applies, then for finite CW-complexes $X$

$$
R^{*}(X) \cong M U^{*}(X) \otimes_{M U^{*}} R .
$$

In general this equality does not extend to infinite $\mathrm{CW}$-complexes since the tensor product on the right-hand side does not necessarily commute with infinite products.

If one omits the compactness condition in the Definition 2.2 and Definition 2.3, then one obtains the axioms for smooth and multiplicative smooth extensions defined on the category of all manifolds. If the coefficients groups $R$ is degree-wise finitely generated (see the corresponding remark in Section 2.2.1), then we obtain the same notion as in [5].

Our construction of the smooth extension of the complex cobordism theory does not depend on any compactness assumption so that there is also a corresponding version of Theorem 2.4 furnishing a multiplicative smooth extension of $(M U, c)$ defined on the category of all smooth manifolds.

2.2.5 We also introduce the notion of a smooth $M U$-orientation (Definition 4.27) of a proper submersion $p: W \rightarrow B$ and define a pushforward $p_{!}: \widehat{M U}(W) \rightarrow \widehat{M U}(B)$ which refines the integration map $p_{!}: M U(W) \rightarrow M U(B)$ (Definition 4.34). In Section 4.4 we show that integration is compatible with the structure maps $a, R, I$ of the smooth extension, functorial, compatible with pullback and the product. We refer to this subsection and Theorem 2.7 for further details. Integration maps play a fundamental role in applications of generalized cohomology theories. This is the case, for example, in the context of T-duality, where we hope to eventually generalize our investigations [6] to a setting in smooth cohomology.

\subsection{Smooth extensions for Landweber exact formal group laws}

2.3.1 If $(R, g)$ is a Landweber exact formal group law, then we let $R^{*}(X):=$ $M U^{*}(X) \otimes_{M U^{*}} R$ denote the associated cohomology theory on finite CW-complexes. We consider the pair $\left(R, c_{R}\right)$, where $c_{R}: R \rightarrow R \otimes_{\mathbb{Z}} \mathbb{R}=: R \mathbb{R}$ is the canonical map.

Theorem 2.5 If $(R, g)$ is a Landweber exact formal group law, then $\left(R, c_{R}\right)$ has a multiplicative smooth extension $\widehat{R}$, given by $\widehat{R}(B)=\widehat{M U}(B) \otimes_{M U^{*}} R$. 
Proof We start with Theorem 2.4 which states that $(M U, c)$ has a multiplicative smooth extension. Since $\Omega^{k}(*)=0$ for $k \neq 0, \Omega^{0}(*) \cong \mathbb{R}$, and $M U^{\text {odd }}=0$, the natural map $\widehat{M U}^{\mathrm{ev}}(*) \rightarrow M U^{\mathrm{ev}}(*)$ is an isomorphism. Hence $\widehat{M U}^{\mathrm{ev}}(*) \cong M U^{*}$, and for a compact manifold $B$ the group $\widehat{M U}(B)$ is an $M U^{*}-$ module. We set $\widehat{R}(B):=\widehat{M U}^{*}(B) \otimes_{M U^{*}} R$ and define the structure maps $R, I, a$ by tensoring the corresponding structure maps for $\widehat{M U}$. Here we identify $R^{*}(B) \cong M U^{*}(B) \otimes_{M U^{*}} R$ and $\Omega(B, R \mathbb{R}) \cong \Omega(B, M U \mathbb{R}) \otimes_{M U^{*}} R$. The only nontrivial point to show is that the sequence

$$
R(B) \stackrel{c_{R}}{\rightarrow} \Omega(B, R \mathbb{R}) / \mathrm{im}(d) \stackrel{a}{\rightarrow} \hat{R}(B) \stackrel{I}{\rightarrow} R(B) \rightarrow 0
$$

is exact. Let us reformulate this as the exactness of

$$
0 \rightarrow \Omega(B, R \mathbb{R}) / c_{R}(R(B)) \rightarrow \widehat{R}(B) \rightarrow R(B) \rightarrow 0 .
$$

We start from the exact sequence

$$
0 \rightarrow \Omega(B, M U \mathbb{R}) / c\left(M U^{*}(B)\right) \rightarrow \widehat{M U}(B) \rightarrow M U^{*}(B) \rightarrow 0 .
$$

Tensoring by $R$ gives

$$
\begin{aligned}
\operatorname{Tor}_{1}^{M U^{*}}\left(M U^{*}(B), R\right) \rightarrow\left(\Omega(B, M U \mathbb{R}) / c\left(M U^{*}(B)\right)\right) \otimes_{M U^{*}} R \\
\rightarrow \widehat{M U}(B) \otimes_{M U^{*}} R \rightarrow M U^{*}(B) \otimes_{M U^{*}} R \rightarrow 0 .
\end{aligned}
$$

Since the tensor product is right exact we have

$$
\left(\Omega(B, M U \mathbb{R}) / c\left(M U^{*}(B)\right)\right) \otimes_{M U^{*}} R \cong \Omega(B, R \mathbb{R}) / c_{R}(R(B)) .
$$

We conclude the exactness of (2-2) from Landweber's Theorem 2.1 which states that $\operatorname{Tor}_{1}^{M U^{*}}\left(M U^{*}(B), R\right) \cong 0$.

2.3.2 Let $p: V \rightarrow A$ be a proper submersion which is smoothly $M U$-oriented (see Definition 4.27) by $o_{p}$. Recall that $\widehat{R}(V)=\widehat{M U}(V) \otimes_{M U_{*}} R$.

Definition 2.6 We define the pushforward map $p_{!}: \hat{R}(V) \rightarrow \widehat{R}(A)$ by $p_{!}(x \otimes z):=$ $p_{!}(x) \otimes z$.

We must show that the pushforward is well defined. Let $u \in M U(*) \cong \widehat{M U}^{\mathrm{ev}}(*)$. We must show that $p_{!}(x \cup u) \otimes z=p_{!} x \otimes u z$. This indeed follows from the special case of the projection formula Lemma 4.39, $p_{!}(x \cup u)=p_{!}(x) \cup u$.

The smooth $M U$-orientation $o_{p}$ of the proper submersion $p$ gives rise to a form $A\left(o_{p}\right) \in \Omega(V, R \mathbb{R})$ which we describe in detail in Lemma 4.29. The next theorem states that the natural and expected properties of a pushforward hold true. 
Theorem 2.7 The following diagram commutes:

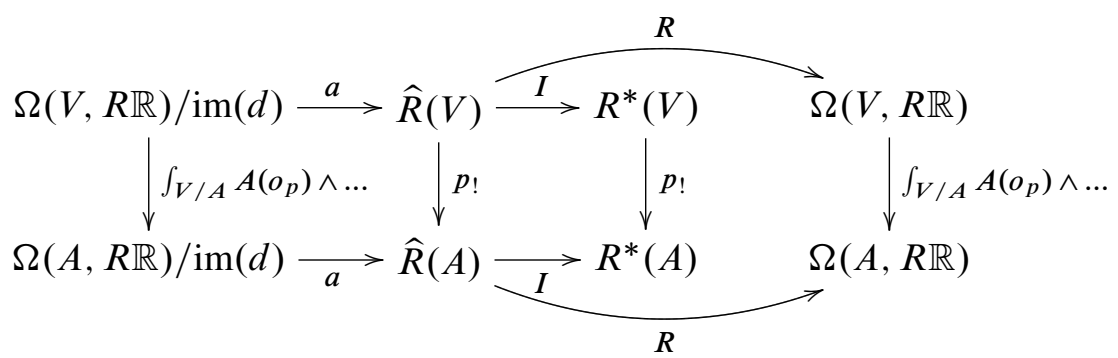

Furthermore, we have the projection formula

$$
p_{!}\left(p^{*} x \cup y\right)=x \cup p_{!} y, \quad x \in \widehat{R}(A), \quad y \in \widehat{R}(V) .
$$

The pushforward is compatible with pullbacks, ie for a Cartesian diagram

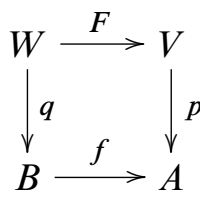

we have

$$
q ! \circ F^{*}=f^{*} \circ p_{!}: \hat{R}(V) \rightarrow \widehat{R}(B),
$$

where $q$ is smoothly $M U$-oriented by $f^{*} o_{p}$.

If $C \rightarrow V$ is a second proper submersion with smooth $M U$-orientation $o_{r}$, then the composition $s:=p \circ r$ has the composed orientation $o_{s}:=o_{p} \circ o_{r}$ (see Definition 4.32), and we have

$$
s_{!}=p_{!} \circ r_{!}: \hat{R}(C) \rightarrow \widehat{R}(A) .
$$

Proof This follows immediately by tensoring with $\mathrm{id}_{R}$ the corresponding results of the pushforward for the extension of $(M U, c)$. These are all proven in Section 4.4.

Corollary 2.8 Let $\left(R_{1}, g_{1}\right)$ and $\left(R_{2}, g_{2}\right)$ be two Landweber exact formal group laws with corresponding cohomology theories $R_{i}(B):=M U(B) \otimes_{M U^{*}} R_{i}$. Let $\phi: R_{1} \rightarrow R_{2}$ be a natural transformation of $M U$-modules. Then $\phi$ lifts to a natural transformation of smooth cohomology theories as in [5, Definition 1.5] or [4, Definition 1.3], $\hat{\phi}(B):=$ $\operatorname{id}_{\widehat{M U}(B)} \otimes \phi$.

In particular, we have a (multiplicative) smooth complex orientation $\widehat{M U}(B) \rightarrow \widehat{K}(B)$ from smooth complex cobordism to smooth $K$-theory.

Here, we use again that $\widehat{K}(B)$ is uniquely determined as a multiplicative extension of $K$-theory [5]. 


\section{Normal $G$-structures and cobordism theories}

\subsection{Representatives of the stable normal bundle}

3.1.1 In the present paper we construct geometric models of smooth extensions of cobordism cohomology theories associated to the families $G(n)$ of classical groups like $U(n), \operatorname{SO}(n), \operatorname{Spin}(n)$, or $\operatorname{Spin}^{c}(n)$. We use the notation $M G(B)$ and are in particular interested in the case where $B$ is a smooth manifold. A cycle for $M G^{n}(B)$ is a proper smooth map $W \rightarrow B$ with a normal $G$-structure such that $\operatorname{dim}(B)-\operatorname{dim}(W)=n$. The relations are given by bordisms.

Cycles for the smooth extension will have in addition a geometric normal $G$-structure. In order to make a precise definition we introduce a rather concrete version of the notion of the stable normal bundle.

3.1.2 Let $X$ be a space or manifold. For $k \in \mathbb{N}$ we denote by $\underline{\mathbb{R}^{k}} X$ the (total space of the) trivial real vector bundle $X \times \mathbb{R}^{k} \rightarrow X$. Let $f: A \rightarrow B$ be a smooth map between manifolds.

Definition 3.1 A representative of the stable normal bundle of $f$ is a real vector bundle $N \rightarrow A$ together with an exact sequence

$$
0 \rightarrow T A \stackrel{(d f, \alpha)}{\longrightarrow} f^{*} T B \oplus \underline{\mathbb{R}}_{A}^{k} \rightarrow N \rightarrow 0,
$$

where we fix only the homotopy class of the projection to $N$.

There is a natural notion of an isomorphism of representatives of stable normal bundles. For an integer $l \in \mathbb{N}$ it is evident how to define the $l$-fold stabilization $N(l):=N \oplus \underline{\mathbb{R}}^{l} A$ as representative of the stable normal bundle with corresponding short exact sequence.

3.1.3 Let $q$ : $C \rightarrow B$ be a smooth map which is transversal to $f$. Then we have a Cartesian diagram

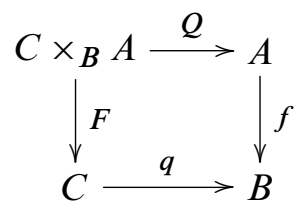

of manifolds. If

$$
0 \rightarrow T A \stackrel{(d f, \alpha)}{\longrightarrow} f^{*} T B \oplus \underline{\mathbb{R}}_{A}^{k} \stackrel{u}{\rightarrow} N \rightarrow 0
$$


represents the stable normal bundle of $f$, then we define the pullback representative of the stable normal bundle of $F$ by

$$
0 \rightarrow T\left(C \times_{B} A\right) \stackrel{(d F, \beta)}{\longrightarrow} F^{*} T C \oplus \underline{\mathbb{R}}^{k} C \times_{B} A \stackrel{\gamma}{\rightarrow} Q^{*} N \rightarrow 0,
$$

with $\beta:=Q^{*} \alpha \circ d Q$ and $\gamma:=Q^{*} u \circ\left(F^{*} d q \oplus \operatorname{id}_{\underline{\mathbb{R}}_{C \times}{ }_{B} A}\right)$. Note that $Q^{*}(N(l)) \cong$ $\left(Q^{*} N\right)(l)$.

3.1.4 We now discuss the stable normal bundle of a composition. Let $g: B \rightarrow C$ a smooth map and

$$
0 \rightarrow T B \stackrel{\stackrel{s}{\leftarrow}}{\stackrel{(d g, \beta)}{\longrightarrow}} g^{*} T C \oplus \underline{\mathbb{R}}_{B}^{l} \stackrel{v}{\rightarrow} M \rightarrow 0
$$

be a representative of the stable normal bundle of $g$. Then we define

$$
0 \rightarrow T A \stackrel{(d(g f), \gamma)}{\longrightarrow}(g f)^{*} T C \oplus \underline{\mathbb{R}}_{A}^{l} \oplus \underline{\mathbb{R}}_{A}^{k} \stackrel{w}{\rightarrow} N \oplus f^{*} M \rightarrow 0
$$

as the associated representative of the stable normal bundle of $g \circ f$. Here $\gamma:=$

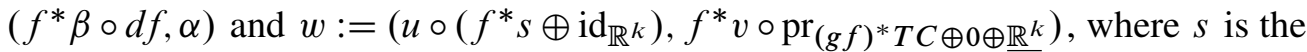
split indicated in (3-1). This split is unique up to homotopy (since the space of such splits is convex) so that the homotopy class of $w$ is well defined.

\section{2 $G$-structures and connections on the stable normal bundle}

3.2.1 Let $G$ be a Lie group with a homomorphism $G \rightarrow \operatorname{GL}(n, \mathbb{R})$ and consider an $n$-dimensional real vector bundle $\xi \rightarrow X$.

Definition 3.2 A $G$-structure on $\xi$ is a pair $(P, \phi)$ of a $G$-principal bundle $P \rightarrow X$ and an isomorphism of vector bundles $\phi: P \times_{G} \mathbb{R}^{n} \stackrel{\sim}{\rightarrow} \xi$.

Definition 3.3 A geometric $G$-structure on $\xi$ is a triple $(P, \phi, \nabla)$, where $(P, \phi)$ is a $G$-structure and $\nabla$ is a connection on $P$.

Note that the trivial bundle $\underline{\mathbb{R}^{n}} X$ has a canonical $G$-structure with $P=X \times G \rightarrow X$.

3.2.2 In order to define a cobordism theory we consider a sequence of groups $G(n)$, $n \in \mathbb{M}$ for an infinite submonoid $\mathbb{M}$ of $\left(\mathbb{N}_{\geq 0},+\right)$ which fit into a chain of commutative diagrams

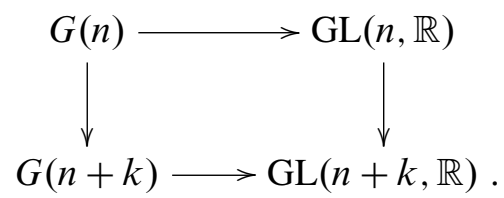


Typically, $\mathbb{M}=\mathbb{N}$ or $\mathbb{M}=2 \mathbb{N}$. This is in particular used in order to define stabilization. In order to define the multiplicative structure we require in addition

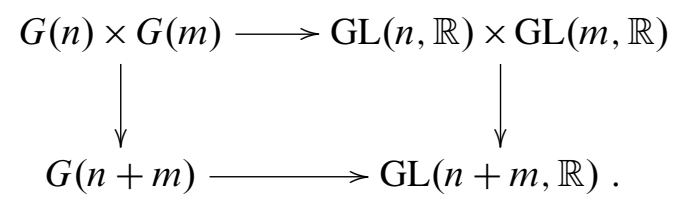

Examples are $O(n), \mathrm{SO}(n), \operatorname{Spin}(n)$, or $\operatorname{Spin}^{c}(n)$. In the present paper we are in particular interested in the complex cobordism theory $M U$. In this case we have $\mathbb{M}=2 \mathbb{N}$ and we set $G(2 n)=U(n)$. By abuse of notation we will use the symbol $G$ to denote such a family of groups, and by $M G$ the corresponding cobordism theory.

3.2.3 Let $f: A \rightarrow B$ be a smooth map between manifold.

Definition 3.4 A representative of a normal $G$-structure on $f$ is given by a pair $(N, P, \phi)$, where $N$ is a representative of the stable normal bundle, and $(P, \phi)$ is a $G(n)$-structure on $N$, where $n:=\operatorname{dim}(N), n \in \mathbb{M}$.

For notational convenience, we write $N$ instead of the short exact sequence with quotient $N$ which is also contained in the data of a representative of the stable normal bundle.

Definition 3.5 A representative of a geometric normal $G$-structure on $f$ is given by a quadruple $(N, P, \phi, \nabla)$, where $N$ is a representative of the stable normal bundle of $f$, and $(P, \phi, \nabla)$ is a geometric $G(n)$-structure on $N$, where $n:=\operatorname{dim}(N), n \in \mathbb{M}$.

There are natural notions of isomorphisms of representatives of normal $G$-structures or geometric normal $G$-structures. In the following we discuss the operations "stabilization", "pullback", and "composition" on the level of representatives of normal $G$-structure and geometric normal $G$-structures.

3.2.4 Let $(N, P, \phi)$ be a representative of a normal $G$-structure on $f: A \rightarrow B$ and consider $l \in \mathbb{M}$. The stabilization $N(l)$ is $N \oplus \underline{\mathbb{R}}^{l} A$. It has a canonical $(G(n) \times G(l))-$ structure with underlying principal bundle $P \times G(l) \rightarrow A$. We get a $G(n+l)$-structure with the underlying principal bundle

$$
P(l):=(P \times G(l)) \times_{G(n) \times G(l)} G(l+n) .
$$

Definition 3.6 We define the stabilization of $(N, P, \phi)$ by

$$
(N, P, \phi)(l):=(N(l), P(l), \phi(l)) .
$$


Let $(N, P, \phi, \nabla)$ is a representative of a geometric normal $G$-structure, then the connection $\nabla$ induces a connection $\nabla(l)$ on $P(l)$.

Definition 3.7 We define the stabilization of $(N, P, \phi, \nabla)$ by

$$
(N, P, \phi, \nabla)(l):=(N(l), P(l), \phi(l), \nabla(l)) .
$$

3.2.5 We now consider the pullback and use the notation introduced in Section 3.1.3. If $(P, \phi)$ is a $G(n)$-structure on $N$, then $\left(Q^{*} P, Q^{*} \phi\right)$ is a $G(n)$-structure on $Q^{*} N$.

Definition 3.8 We define the pullback of a normal $G$-structure by

$$
q^{*}(N, P, \phi):=\left(Q^{*} N, Q^{*} P, Q^{*} \phi\right) .
$$

Definition 3.9 We define the pullback of a geometric normal $G$-structure by

$$
q^{*}(N, P, \phi, \nabla):=\left(Q^{*} N, Q^{*} P, Q^{*} \phi, Q^{*} \nabla\right) .
$$

3.2.6 We now discuss the composition. Continuing with the notation of Section 3.1.4 we consider

$$
A \stackrel{f}{\rightarrow} B \stackrel{g}{\rightarrow} C
$$

and representatives of normal $G$-structures $(N, P, \phi)$ and $(M, Q, \psi)$ on $f$ and $g$. The sum $N \oplus f^{*} M$ has a natural $G(n) \times G(m)$-structure with underlying $(G(n) \times G(m))$ bundle $P \times_{A} f^{*} Q$, and therefore a $G(n+m)$-structure with underlying bundle

$$
R:=\left(P \times_{A} f^{*} Q\right) \times_{G(n) \times G(m)} G(n+m)
$$

with isomorphism $\rho: R \times_{\mathrm{GL}(n+m)} \mathbb{R}^{n+m} \cong N \oplus f^{*} M$.

Definition 3.10 We define the composition of representatives of normal $G$-structures by

$$
(M, Q, \psi) \circ(N, P, \phi):=\left(N \oplus f^{*} M, R, \rho\right) .
$$

If $\nabla^{P}$ and $\nabla^{Q}$ are connections on $P$ and $Q$, then we get an induced connection $\nabla^{R}$ on $R$.

Definition 3.11 We define the composition of representatives of geometric normal $G$-structures by

$$
(M, Q, \psi, \nabla) \circ(N, P, \phi, \nabla):=\left(N \oplus f^{*} M, R, \rho, \nabla^{R}\right) .
$$


3.2.7 The following assertions are obvious.

Lemma 3.12 (1) On the level of representatives of normal $G$-structures or geometric normal $G$-structures, pullback and composition commute with stabilization.

(2) On the level of representatives of normal $G$-structures or geometric normal $G$-structures, pullback and composition are functorial.

(3) On the level of representatives of normal $G$-structures or geometric normal $G$-structures, pullback and composition commute with each other.

\subsection{A cycle model for $M G$}

3.3.1 Let us fix a family of groups $G$ and $\mathbb{M}$ as in Section 3.2.2. It determines a multiplicative cohomology theory which is represented by a Thom spectrum MG. The map $G(n) \rightarrow \operatorname{GL}(n, \mathbb{R})$ induces a map of classifying spaces $B G(n) \rightarrow B G L(n, \mathbb{R})$. Let $\xi_{n} \rightarrow B G(n)$ denote the pullback of the universal $\mathbb{R}^{n}$-bundle. Then for $n \in \mathbb{M}$ we define $\mathbf{M G}^{n}:=B G(n)^{\xi_{n}}$, where for a vector bundle $\xi \rightarrow X$ we write $X^{\xi}$ for its Thom space. The family of spaces $\mathbf{M G}^{n}, n \geq 0$, fits into a spectrum with structure maps

$$
\Sigma^{d} \mathbf{M G}^{n} \cong B G(n)^{\xi_{n} \oplus \underline{\mathbb{R}}^{d} B G(n)} \rightarrow B G(n+d)^{\xi_{n+1}} \cong \mathbf{M G}^{n+d}, \quad n, n+d \in \mathbb{M}
$$

where we use the canonical Cartesian diagram

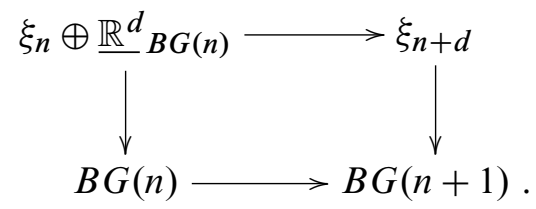

The ring structure is induced by

$\mathbf{M G}^{n} \wedge \mathbf{M G}^{m} \cong B G(n)^{\xi_{n}} \wedge B G(m)^{\xi_{m}} \cong(B G(n) \times B G(m))^{\xi_{n} \boxplus \xi_{m}}$

$$
\rightarrow B G(n+m)^{\xi_{n+m}} \cong \mathbf{M G}^{n+m},
$$

for $n, m \in \mathbb{M}$, using the canonical Cartesian diagram

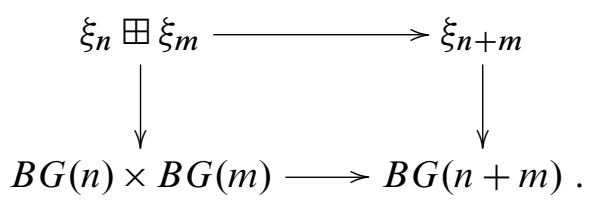


For $l \notin \mathbb{M}$ we set $M G^{l}:=\Sigma^{l-d} M G^{d}$, where $d \leq l$ is maximal with $d \in \mathbb{M}$. The corresponding structure maps and multiplication maps are given as suspensions of the maps described above.

If $A$ is a manifold (or more generally a finite $\mathrm{CW}$-complex), then the homotopy theoretic definition of the cobordism cohomology group is

$$
\mathbf{M G}^{n}(A):=\lim _{k}\left[\Sigma^{k} A_{+}, \mathbf{M G}^{n+k}\right],
$$

where the limit is taken over the stabilization maps

$$
\left[\Sigma^{k} A_{+}, \mathbf{M G}^{n+k}\right] \rightarrow\left[\Sigma \Sigma^{k} A_{+}, \Sigma \mathbf{M G}^{n+k}\right] \rightarrow\left[\Sigma^{k+1} A_{+}, \mathbf{M G}^{n+k+1}\right],
$$

and $A_{+}$is the union of $A$ and a disjoint base point. Temporarily we use the boldface notation of the homotopy theoretic definition of the cobordism cohomology theory. For details we refer to Switzer [23] or Stong [22].

3.3.2 We now present a cycle model of the $G$-cobordism theory. Let $A$ be a smooth manifold.

Definition 3.13 A precycle $(p, v)$ of degree $n \in \mathbb{Z}$ over $A$ consists of a smooth map $p: W \rightarrow A$ from a smooth manifold $W$ of dimension $\operatorname{dim}(W)=\operatorname{dim}(A)-n$, and a representative $v$ of a normal $G$-structure on $p$ (see Definition 3.4). A cycle of degree $n \in \mathbb{Z}$ over $A$ is a precycle $(p, v)$ of degree $n$, where $p$ is proper.

There is a natural notion of an isomorphism of precycles.

3.3.3 Let $c:=(p, v)$ be a precycle over $A$ and $q: B \rightarrow A$ be transverse to $p$.

Definition 3.14 We define the pullback $q^{*} c:=\left(q^{*} p, q^{*} v\right)$, a precycle over $B$.

The pullback is functorial by Lemma 3.12.

3.3.4 We now consider precycles $c=(p, v)$ over $A$ and $d=(q, \mu)$ over $C$ with underlying maps $p: B \rightarrow A$ and $q: A \rightarrow C$.

Definition 3.15 We define the composition

$$
d \circ c:=(q \circ p, \mu \circ v)
$$

using Definition 3.10.

The composition $d \circ c$ is a precycle over $C$. The composition is associative and compatible with pullback. 
3.3.5 Let $c:=(p, v), p: W \rightarrow A$, and $d:=(q, \mu), q: V \rightarrow B$ be precycles over $A$ and $B$. Then we can form the diagram:

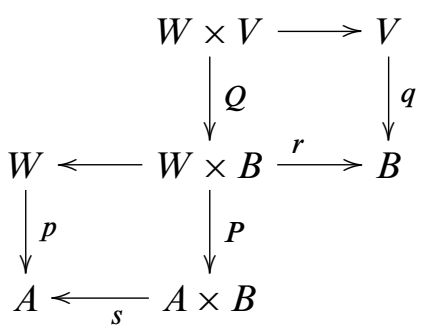

Definition 3.16 We define the product of the precycles $c$ and $d$ to be the precycle $c \times d:=s^{*} c \circ r^{*} d$ over $A \times B$.

Note that there is an equivalent definition based on the diagram:

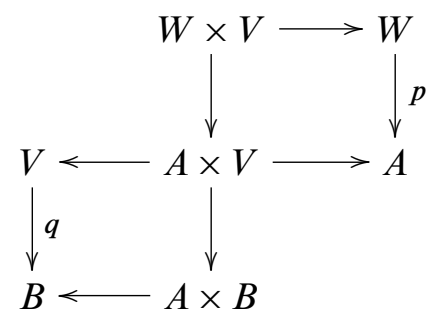

It follows from the functoriality of the composition and its compatibility with the pullback that the product of precycles is associative.

3.3.6 We consider a precycle $b:=((f, p), \nu)$ over $\mathbb{R} \times A$.

Definition 3.17 The precycle $b$ is called a bordism datum if $f$ is transverse to $\{0\} \in \mathbb{R}$ and $\left.p\right|_{\{f \geq 0\}}$ is proper. We define the precycle $\partial b:=i^{*} b$, where $i: A \rightarrow \mathbb{R} \times A$, $i(a):=(0, a)$.

3.3.7 Let $c=(p, v)$ be a precycle and $l \in \mathbb{N}$.

Definition 3.18 We define the $l$-fold stabilization of the precycle $c$ by $c(l):=$ $(p, v(l))$ (see Definition 3.6). 
3.3.8 We now come to the geometric picture of the cobordism theory $M G$. We consider a smooth manifold $A$ and let $Z M G(A)$ denote the semigroup of isomorphism classes of cycles over $A$ with respect to disjoint union. Recall that a relation $\sim$ on a semigroup is compatible with the semigroup structure if $a \sim b$ implies that $a+c \sim b+c$ for all $c$.

Definition 3.19 Let " " be the minimal equivalence relation which is compatible with the semigroup structure and satisfies:

(1) If $b$ is a bordism datum, then $\partial b \sim 0$.

(2) If $l \in \mathbb{N}$, then $c(l) \sim c$.

We let $M G(A):=Z M G(A) / \sim$ denote the quotient semigroup.

3.3.9 Let 0 denote the cycle of degree $n$ given by the empty manifold. The following Lemma will be useful in calculations.

Lemma 3.20 Let $c$ be a cycle which is equivalent to 0 . Then there exists a bordism datum $b$ and $l \geq 0$ such that $c(l) \cong \partial b$.

We leave the proof to the interested reader.

3.3.10 We now describe the functoriality, the product, orientations, and the integration on the level of cycles.

(1) (Functoriality) Let $f: B \rightarrow A$ be a smooth map and $x \in M G(A)$. We can represent $x$ by a cycle $c=(p, v)$ such that $p$ and $f$ are transverse. Then $f^{*} x$ is represented by $f^{*} c$.

(2) (Product) Let $c$ and $d$ be cycles for $x \in M G^{n}(A)$ and $y \in M G^{m}(B)$. Then $x \times y \in M G^{n+m}(A \times B)$ is represented by the cycle $c \times d$ (see Definition 3.16). We get the interior product using the pullback along the diagonal.

(3) (Integration) Let $d$ be a cycle over $A$ with underlying map $q: V \rightarrow A$. In this situation we have an integration map

$$
q !: M G(V) \rightarrow M G(A) .
$$

If $x \in M G(V)$ is represented by the cycle $c$, then $q !(x)$ is represented by the cycle $d \circ c$ (see Definition 3.15). 
(4) (Suspension) Let $i: * \rightarrow S^{1}$ denote the embedding of a point. For each $d \in \mathbb{M}$, the trivial bundle $\underline{\mathbb{R}}^{d} *$ represents the stable normal bundle which of course has a canonical $G(d)$-structure. In this way $i$ is the underlying map of a cycle $\{i\} \in Z M G^{1}\left(S^{1}\right)$ which represents a class $[i] \in M G^{1}\left(S^{1}\right)$.

For a manifold $A$ we define $M G^{k}(A) \rightarrow M G^{k+1}\left(S^{1} \times A\right), x \mapsto\{i\} \times x$, which on the level of cycles is represented by $c \mapsto\{i\} \times c$. This transformation is essentially the suspension morphism (not an isomorphism, since we neither use reduced cohomology nor the suspension of $A$ ).

3.3.11 In order to show that the operations defined above on the cycle level descend through the equivalence relation $\sim$ the following observations are useful. Let $b=$ $((f, p), \mu)$ be a bordism datum over $A$ with underlying map $(f, p): W \rightarrow \mathbb{R} \times A$. Assume that $q: B \rightarrow A$ is transverse to $p$ and $\left.p\right|_{\{f=0\}}$. Then we can form the bordism datum $\left(\operatorname{id}_{\mathbb{R}} \times q\right)^{*} b$ over $B$ which will be denoted by $q^{*} b$. Note that

$$
q^{*} \partial b \cong \partial q^{*} b
$$

Let $e$ be a cycle over $B$. Then we can form $b \times e$ which we can interpret as a bordism datum over $B \times A$. Note that

$$
\partial(b \times e) \cong \partial b \times e .
$$

Let $d$ be a cycle with underlying map $A \rightarrow B$. Let pr: $\mathbb{R} \times B \rightarrow B$ be the projection. Then we can form the bordism datum $\operatorname{pr}^{*} d \circ b$ over $B$. Note that

$$
\partial\left(\mathrm{pr}^{*} d \circ b\right) \cong d \circ \partial b
$$

Finally, if $c$ is a cycle over $W$, then we can form the bordism datum $b \circ c$ over $B$, and have

$$
\partial(b \circ c) \cong \partial b \circ c
$$

3.3.12 We now have a geometric and a homotopy theoretic picture of the $G$-cobordism theory which we distinguish at the moment by using roman and boldface letters.

Proposition 3.21 There is an isomorphism of ring-valued functors $M G(A) \cong \mathbf{M G}(A)$ on compact manifolds. This isomorphism preserves the product and is compatible with pushforward.

Proof This follows from the Pontryagin-Thom construction. Since this construction for cobordism cohomology (as opposed to homology) seems not to be so well known 
let us shortly indicate the main ideas. For concreteness let us consider the case of complex cobordism $M U$ and even $2 n$. We have

$$
M U^{2 n}(A) \cong \operatorname{colim}_{i}\left[\Sigma^{2 i} A, M U^{2 n+2 i}\right] .
$$

Let $h: \Sigma^{2 i} A \rightarrow M U^{2 n+2 i}$ represent some class in $M U^{2 n}(A)$. Recall that $M U^{2 n+2 i}=$ $B U(n+i)^{\xi_{n+i}}$ is the Thom space the universal bundle $\xi_{n+i} \rightarrow B U(n+i)$. The latter is itself the colimit of Thom spaces

$$
B U(n+i)^{\xi_{n+i}} \cong \operatorname{colim}_{k} \operatorname{Gr}_{n+i}\left(\mathbb{C}^{n+i+k}\right)^{\xi_{n+i}}
$$

of tautological bundles $\xi_{n+i}$ over the Grassmannians $\mathrm{Gr}_{n+i}\left(\mathbb{C}^{n+i+k}\right)$ of $(n+i)-$ dimensional subspaces in $\mathbb{C}^{n+i+k}$. We can assume that $h$ factors over some Thom space $\mathrm{Gr}_{n+i}\left(\mathbb{C}^{n+i+k}\right)^{\xi_{n+i}}$, and that the induced map

$$
S^{2 i} \times A \stackrel{p}{\rightarrow} \Sigma^{2 i} A \stackrel{f}{\rightarrow} \mathrm{Gr}_{n+i}\left(\mathbb{C}^{n+i+k}\right)^{\xi_{n+i}}
$$

is smooth and transverse to the zero section of $\xi_{n+i}$, where $p$ is the canonical projection. The preimage of the zero section is a submanifold $W \subset S^{2 i} \times A$ of codimension $2 n+2 i$. We let $f: W \rightarrow A$ be induced by the projection. We use the standard embedding $S^{2 i} \rightarrow \mathbb{R}^{2 i+2}$ in order to trivialize the bundle $T S^{2 i} \oplus \mathbb{R}_{S^{2 i}} \cong S^{2 i} \times \mathbb{R}^{2 i+2}$. The embedding $W \hookrightarrow S^{2 i} \times A$ thus induces naturally an embedding

$$
\left.\left.T W \rightarrow T\left(A \times S^{i}\right)\right|_{W} \cong f^{*} T A \oplus T S^{2 i}\right|_{W} \rightarrow f^{*} T A \oplus \underline{\mathbb{R}^{2 i+2}} W .
$$

Moreover, the differential of $h$ identifies the normal bundle $N:=f^{*} T A \oplus \underline{\mathbb{R}^{2 i+2}} W / T W$ with the pullback $\left.h\right|_{W} ^{*} \xi_{n+i} \oplus \mathbb{C}_{W}$, which has a canonical complex structure. In this way we get the normal bundle sequence

$$
0 \rightarrow T W \rightarrow f^{*} T A \oplus \underline{\mathbb{R}^{2 i+2}} M \rightarrow N \rightarrow 0
$$

and the $U$-structure $v=(N, P, \phi)$ on $N$. Note that $f: W \rightarrow A$ is proper so that we get a cycle $(f, v)$ of degree $n$. One now proceeds as in the case of bordism homology and shows that the class $[f, v] \in M U^{2 n}(A)$ only depends on the class $[h] \in M U^{2 n}(A)$. In this way we get a map $M U^{2 n}(A) \rightarrow M U^{2 n}(A)$.

Conversely one starts with a cycle $(f, v)$ of degree $n$. One observes that up to stabilization and homotopy the normal bundle sequence

$$
0 \rightarrow T W \rightarrow f^{*} T A \oplus \underline{\mathbb{R}}_{W}^{k} \rightarrow N \rightarrow 0
$$

comes from an embedding of $i: W \hookrightarrow S^{k-1} \times A$ such that $f=\operatorname{pr}_{A} \circ i$. Then we let $W \rightarrow B U(n+(n+k) / 2)$ be a classifying map of $N$ (necessarily, $\operatorname{dim}_{\mathbb{R}} N=n+k$ is even). It gives rise to a map of Thom spaces $W^{N} \rightarrow B U(n+i)^{\xi_{(n+k) / 2}}$. We finally 
precompose with the clutching map $\Sigma^{k} A \rightarrow W^{N}$ in order to get a map $h: \Sigma^{k} A \rightarrow$ $M U^{n+k}$.

One checks that this construction gives the inverse map $M U^{n}(A) \rightarrow M U^{n}(A)$. A further standard argument checks that these maps are compatible with the abelian group and ring structures and the pushforward.

In view of Proposition 3.21 we can drop the boldface notation for the homotopy theoretic cobordism.

\subsection{Power series and genera}

3.4.1 The basic datum for a multiplicative smooth extension of a generalized cohomology theory $h$ is a pair $(h, c)$, where $c: h \rightarrow H R$ is a natural transformation from $h$ into the ordinary cohomology with coefficients in a graded ring $R$ over $\mathbb{R}$. The transformation $c$ induces in particular a homomorphism of coefficients $h^{*} \rightarrow R^{*}$. Our construction of smooth extensions of cobordism theories is based on a description of $c$ in terms of characteristic numbers of stable normal bundles.

A ring homomorphisms $c: M G^{*} \rightarrow R^{*}$ is called a $G$-genus. One can classify SO and $U$-genera in terms of formal power series (see Hirzebruch, Berger and Jung [13] and Theorem 3.22). Genera for other cobordism theories can be derived from transformations like $M$ Spin $\rightarrow M S O$. Since the details in the real and complex case differ slightly, in the present paper we restrict to our main example $G:=M U$, ie $\mathbb{M}=2 \mathbb{N}_{\geq 0}$, $G(2 n)=U(n)$. It is easy to modify the constructions for other cases like $M \operatorname{Spin}^{c}$, $M \mathrm{SO}$ or $\mathrm{Spin}^{c}$.

3.4.2 Let $R$ be a commutative $\mathbb{Z}$-graded algebra over $\mathbb{R}$ with $1 \in R^{0}$. By $R \llbracket z \rrbracket$ we denote the graded ring of formal power series, where $z$ has degree 2. Let $\phi \in R \llbracket z \rrbracket^{0}$ be a power series of the form $1+\phi_{1} z+\phi_{2} z^{2}+\cdots$ (note that $\operatorname{deg}\left(\phi_{i}\right)=-2 i$ ). To such a power series we associate a genus $r_{\phi}: M U^{*} \rightarrow R^{*}$ as in [18, Section 19].

Theorem 3.22 [13] The correspondence $\phi \rightarrow r_{\phi}$ gives a bijection between the set $R \llbracket z \rrbracket^{0}$ and $R$-valued $U$-genera.

In the following we describe the associated natural transformation $r_{\phi}: M U(A) \rightarrow$ $H(A, R)$ of cohomology theories on the level of cycles, following the procedure as described in [18]. 
3.4.3 We define the power series $K_{\phi} \in R \llbracket \sigma_{1}, \sigma_{2}, \ldots, \rrbracket^{0}$ (where $\sigma_{i}$ has degree $2 i$ ) such that

$$
K_{\phi}\left(\sigma_{1}, \sigma_{2}, \ldots\right)=\prod_{i=1}^{\infty} \phi\left(z_{i}\right)
$$

holds if we replace $\sigma_{i}$ by the elementary symmetric functions $\sigma_{i}\left(z_{1}, \ldots\right)$.

3.4.4 Note that $G(2 k)=U(k)$ (see Section 3.2.2). Let $N \rightarrow W$ be an $n$-dimensional real vector bundle for $n$ even with a $G(n)$-structure $(P, \phi)$. Then we have Chern classes $c_{j}(N):=c_{j}(P) \in H^{2 j}(W, \mathbb{R})$.

Definition 3.23 We define the characteristic class

$$
\phi(N):=K_{\phi}\left(c_{1}(N), c_{2}(N), \ldots\right) \in H^{0}(A, R) .
$$

The following properties are well-known (see Hirzebruch, Berger and Jung [13]).

Lemma 3.24 (1) Let $\underline{\mathbb{R}}_{A}$ have the trivial $G(k)$-structure. Then $\phi\left(\underline{\mathbb{R}^{k}}\right)_{A}=1$ for all $k \geq 0$.

(2) If $M$ is a second bundle with a $G(m)$-structure, and $N \oplus M$ has the induced $G(n+m)$-structure, then we have $\phi(N \oplus M)=\phi(N) \cup \phi(M)$.

(3) If $f: B \rightarrow A$ is a continuous map, then we have $f^{*} \phi(N)=\phi\left(f^{*} N\right)$, if we equip $f^{*} N$ with the induced $G(n)$-structure.

3.4.5 Consider a cycle $c=(p, v) \in Z M U(A)$ of degree $n$ with underlying map $p: W \rightarrow A$ and normal $U$-structure $v=(N, P, \phi)$. Then $p$ is a proper map which is oriented for the ordinary cohomology theory $H R$. In particular, we have an integration $p_{!}: H^{*}(W, R) \rightarrow H^{*+n}(A, R)$.

Definition 3.25 We define

$$
\widetilde{r}_{\phi}(c):=p_{!}(\phi(N)) \in H^{n}(A, R) .
$$

3.4.6 The following Lemma implies half of Theorem 3.22. What is missing is the argument that every $R$-valued $U$-genus comes from a formal power series.

Lemma 3.26 The map $\widetilde{r}_{\phi}$ descends through $\sim$ and induces a natural transformation $r_{\phi}: M U(A) \rightarrow H(A, R)$ of ring-valued functors. 
Proof Using the first and second property in Lemma 3.24 one checks that $\widetilde{r}_{\phi}(c)=$ $\tilde{r}_{\phi}(c(l))$.

Assume that $b=((f, q), \mu)$ with underlying map $(f, q): W \rightarrow \mathbb{R} \times A$ and $\mu=$ $(M, Q, \lambda)$ is a bordism datum. Then we get the Cartesian diagram

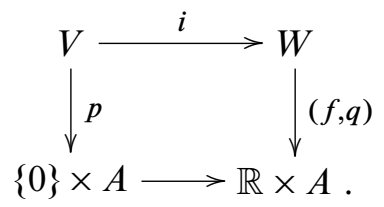

Set $N:=i^{*} M$. Therefore $p_{!}(\phi(N))=p_{!}\left(\phi\left(i^{*} M\right)\right)=p_{!}\left(i^{*} \phi(M)\right)=0$ by the bordism invariance of the pushforward in ordinary cohomology and the third property of Lemma 3.24. Thus the transformation $r_{\phi}$ is well defined.

It is natural since for $f: B \rightarrow A$ which is transverse to $p$ we have a Cartesian diagram

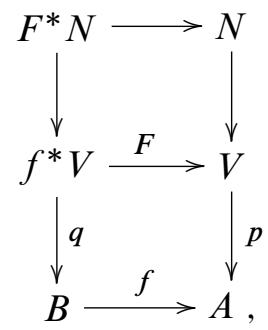

the bundle $F^{*} N$ represents the stable normal bundle of $q$, and

$$
q_{!}\left(\phi\left(F^{*} N\right)\right)=q_{!}\left(F^{*} \phi(N)\right)=f^{*} p_{!}(\phi(N))
$$

by the projection formula. This implies that $f^{*} \widetilde{r}_{\phi}(c)=\widetilde{r}_{\phi}\left(f^{*} c\right)$.

We claim that the transformation is also multiplicative. To this end we consider a cycle $d=(q, \mu)$ with underlying map $q: V \rightarrow B$ and normal $G$-structure $\mu=(M, Q, \lambda)$. Then the underlying proper map of $c \times d \in Z M U(A \times B)$ is $p \times q: W \times V \rightarrow A \times B$, and the bundle $N \boxplus M$ represents its normal $G$-structure. We thus have

$$
(p \times q) !(\phi(N \boxplus M))=(p \times q) !(\phi(N) \times \phi(M))=p_{!}(\phi(N)) \times q !(\phi(M)) .
$$

This implies

$$
\tilde{r}_{\phi}(c \times d)=\tilde{r}_{\phi}(c) \times \widetilde{r}_{\phi}(d) .
$$


3.4.7 The most important example for the present paper is given by the ring $M U \mathbb{R}:=$ $M U^{*} \otimes_{\mathbb{Z}} \mathbb{R}$. The $M U^{*}$-module $M U \mathbb{R}$ is Landweber exact. Hence, for a compact manifold or finite CW-complex $A$ we have $H^{*}(A, M U \mathbb{R}) \cong M U^{*}(A) \otimes_{M U^{*}} M U \mathbb{R}$ and therefore a canonical natural transformation $r: M U^{*}(A) \rightarrow H^{*}(A, M U \mathbb{R}), x \mapsto x \otimes 1$. This transformation is a genus $r=r_{\phi}$ for a certain power series $\phi \in M U \mathbb{R} \llbracket x \rrbracket^{0}$. We refer to [13] for further details on $\phi$.

\section{The smooth extension of $M U$}

\subsection{Characteristic forms}

4.1.1 Let $\phi \in R \llbracket z \rrbracket^{0}$ be as in Section 3.4.2 and $G$ be the family of groups Section 3.2.2 associated to $U(n), n \geq 0$.. We first lift the construction of the characteristic class $\phi(N) \in H^{0}(A, R)$ of vector bundles $N \rightarrow A$ with $G(n)$-structure to the form level.

Let $\left(P, \psi, \nabla^{N}\right)$ be a geometric $G(n)$-structure on $N \rightarrow A$. By $R^{\nabla^{N}} \in \Omega^{2}(A$, $\operatorname{End}(N))$ we denote the curvature of the connection $\nabla^{N}$. The fiberwise polynomial bundle morphism det: $\operatorname{End}(N) \rightarrow \underline{\mathbb{R}}_{A}$ extends to det: $\Omega^{\mathrm{ev}}(A, \operatorname{End}(N)) \rightarrow \Omega^{\mathrm{ev}}(A)$. As usual we define the Chern forms $c_{i}\left(\nabla^{N}\right) \in \Omega^{2 i}(A)$ by

$$
1+c_{1}\left(\nabla^{N}\right)+c_{2}\left(\nabla^{N}\right)+\cdots=\operatorname{det}\left(1+\frac{1}{2 \pi i} R^{\nabla^{N}}\right) .
$$

Definition 4.1 If $N \rightarrow A$ is a real vector bundle with a geometric $G(n)$-structure, then we define

$$
\phi\left(\nabla^{N}\right):=K_{\phi}\left(c_{1}\left(\nabla^{N}\right), c_{2}\left(\nabla^{N}\right), \ldots\right) \in \Omega^{0}(A, R) .
$$

4.1.2 The properties stated in Lemma 3.24 lift to the form level by well-known properties of the Chern-Weil calculus.

Lemma 4.2 (1) Let $k \geq 0$ and $\underline{\mathbb{R}}_{A}^{k}$ have the trivial $G(k)$-structure with the trivial connection. Then we have $\phi\left(\nabla \frac{\mathbb{R}^{k}}{A}\right)=1$.

(2) If $M \rightarrow A$ is a second bundle with a geometric $G(m)$-structure and assume that $N \oplus M$ has the induced geometric $G(n+m)$-structure, then we have $\phi\left(\nabla^{N \oplus M}\right)=\phi\left(\nabla^{N}\right) \wedge \phi\left(\nabla^{M}\right)$.

(3) Assume that $f: B \rightarrow A$ is a smooth map. Then we have $f^{*} \phi\left(\nabla^{N}\right)=\phi\left(\nabla^{*} N\right)$, if we equip $f^{*} N$ with the induced geometric $G(n)$-structure. 
4.1.3 We now define the cycles for our cohomology groups.

Definition 4.3 A geometric precycle over $A$ is a pair $(p, v)$ of a smooth map $p: V \rightarrow$ $A$ and a geometric normal $G$-structure $v$ (see Definition 3.5). A geometric precycle is a cycle if $p$ is proper.

Usually we will denote geometric precycles by $\tilde{c}$, where $c$ denotes the underlying precycle. Since a principal bundle always admits connections, every precycle can be refined to a geometric precycle. If $v=(N, P, \phi, \nabla)$, then we will write $\nabla^{v}:=\nabla$.

4.1.4 Let $\Omega_{-\infty}(A):=C^{-\infty}\left(A, \Lambda^{*} T^{*} A\right)$ denote the differential forms with distributional coefficients. We identify this space with the topological dual of the space $C_{c}^{\infty}\left(A, \Lambda^{n-*} T^{*} A \otimes \Lambda_{A}\right)$, where $\Lambda_{A} \rightarrow A$ is the real orientation bundle and $n=$ $\operatorname{dim}(A)$. For this identification we use cup product and integration of an $n$-form with values in the orientation bundle over $A$. Finally, we define $\Omega_{-\infty}(A, R):=$ $\Omega_{-\infty}(A) \otimes_{\mathbb{R}} R$ using the algebraic tensor product.

A morphism of complexes inducing an isomorphism in cohomology is called a quasiisomorphism. It is well-known (see de Rham [20], or do this exercise using Lemma 4.11) that the inclusion $\Omega(A) \hookrightarrow \Omega_{-\infty}(A)$ is a quasi-isomorphism. Hence, $\Omega(A, R) \hookrightarrow$ $\Omega_{-\infty}(A, R)$ is a quasi-isomorphism, too.

4.1.5 Let $p: V \rightarrow A$ be a proper smooth oriented map. The orientation of $p$ gives an isomorphism $p^{*} \Lambda_{A} \stackrel{\sim}{\rightarrow} \Lambda_{V}$. We then define the pushforward

$$
p_{!}: \Omega_{-\infty}(V) \rightarrow \Omega_{-\infty}(A)
$$

of degree $\operatorname{dim}(A)-\operatorname{dim}(V)$ by the formula

$$
\langle p ! \omega, \sigma\rangle=\left\langle\omega, p^{*} \sigma\right\rangle, \quad \omega \in \Omega_{-\infty}(V), \sigma \in \Omega\left(A, \Lambda_{A}\right)
$$

holds true. By tensoring with the identity of $R$ we get the map $p_{!}: \Omega_{-\infty}(V, R) \rightarrow$ $\Omega_{-\infty}(A, R)$. Stokes' theorem implies

$$
p_{!} \circ d=d \circ p_{!} .
$$

We get an induced map in cohomology such that the following diagram commutes:

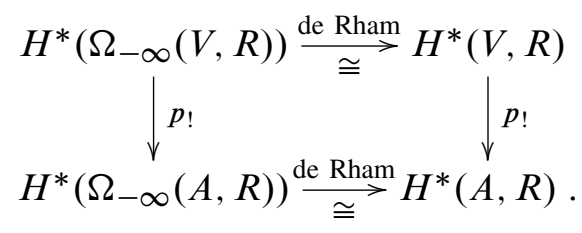


4.1.6 Let $\tilde{c}=(p, v)$ be a geometric cycle of degree $n$.

Definition 4.4 We define $T(\widetilde{c}):=p_{!}\left(\phi\left(\nabla^{v}\right)\right) \in \Omega_{-\infty}^{n}(A, R)$.

This form is closed, and by (4-1) we have the following equality in de Rham cohomology:

$$
[T(\widetilde{c})]=p_{!}(\phi(N))=\widetilde{r}_{\phi}(c) .
$$

4.1.7 We now consider a bordism datum $b=((f, q), \mu)$ over a manifold $A$ with $(f, q): W \rightarrow \mathbb{R} \times A$. We build the composition

$$
q ! \circ \chi_{\{f \geq 0\}}: \Omega^{k}(W) \rightarrow \Omega_{-\infty}^{k+l}(A),
$$

where $l:=\operatorname{dim}(A)-\operatorname{dim}(W)$, and $\chi_{U}$ is the multiplication operation with the characteristic function of the subset $U$. Stokes' theorem implies in this case that

$$
d \circ q_{!} \circ \chi_{\{f \geq 0\}}-q ! \circ \chi_{\{f \geq 0\}} \circ d=\left(q_{0}\right) ! \circ i^{*},
$$

where $q_{0}: W_{0} \rightarrow A$ is defined by the Cartesian diagram

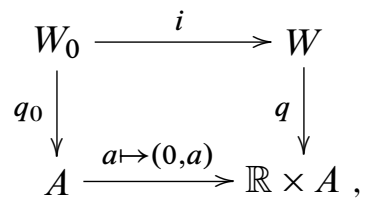

ie $q_{0}$ is the underlying map of $\partial b$.

Definition 4.5 Let $\tilde{b}:=((f, q), \widetilde{v})$ be a geometric refinement of $b$. We define

$$
T(\tilde{b}):=q ! \circ \chi_{\{f \geq 0\}}\left(\phi\left(\nabla^{\widetilde{v}}\right)\right) \in \Omega_{-\infty}(A) .
$$

Equation (4-3) shows that

$$
d T(\tilde{b})=T(\partial \tilde{b})
$$

\subsection{The smooth extension of $M U$}

4.2.1 In the present subsection we construct the smooth extension associated to the pair $\left(M U, r_{\phi}\right)$, where $\phi \in R \llbracket z \rrbracket^{0}$ is as in Section 3.4.2, and $r_{\phi}$ is the associated natural transformation $M U(A) \rightarrow H(A, R)$. Recall the notions of a cycle and a geometric cycle from Definition 3.13 and Definition 4.3. The cycles for the smooth extension $\widehat{M U}$ of $M U$ will be called smooth cycles. 
Definition 4.6 A smooth cycle of degree $n$ is a pair $\widehat{c}:=(\widetilde{c}, \alpha)$, where $\tilde{c}$ is a geometric cycle of degree $n$, and $\alpha \in \Omega_{-\infty}^{n-1}(A, R) / \operatorname{im}(d)$ is such that

$$
T(\widetilde{c})-d \alpha:=\Omega(\widehat{c}) \in \Omega^{n}(A, R) .
$$

The point here is that $T(\widetilde{c})-d \alpha$ is a smooth representative of the cohomology class represented by $T(\widetilde{c})$. The latter is in general a singular form. To be explicit note that in the definition above,

$$
\operatorname{im}(d):=\operatorname{im}\left(d: \Omega_{-\infty}^{n-2}(A, R) \rightarrow \Omega_{-\infty}^{n-1}(A, R)\right),
$$

ie we allow differentials of forms with distribution coefficients.

4.2.2 There is an evident notion of an isomorphism of smooth cycles. We form the graded semigroup $Z \widehat{M U}(A)$ of isomorphism classes of smooth cycles such that the sum is given by

$$
(\widetilde{c}, \alpha)+\left(\widetilde{c}^{\prime}, \alpha^{\prime}\right)=\left(\widetilde{c}+\widetilde{c}^{\prime}, \alpha+\alpha^{\prime}\right),
$$

where, as in the nongeometric case, $\tilde{c}+\tilde{c}^{\prime}$ is given by the disjoint union.

4.2.3 We will define the smooth cobordism group $\widehat{M U}(A)$ as the quotient of $Z \widehat{M U}(A)$ by an equivalence relation generated by stabilization and bordism.

Definition 4.7 Let " " be the minimal equivalence relation on $Z \widehat{M U}(A)$ which is compatible with the semigroup structure (see Section 3.3.8) and such that:

(1) For $l \in \mathbb{M}$ we have $(\tilde{c}, \alpha) \sim(\widetilde{c}(l), \alpha)$, where $\tilde{c}(l)$ is the $l$-fold stabilization defined by $(p, v)(l):=(p, v(l))$ (see Definition 3.7).

(2) For a geometric bordism datum $\tilde{b}$ we have $(\partial \tilde{b}, T(\tilde{b})) \sim 0$.

We define $\widehat{M U}^{n}(A):=Z \widehat{M U}^{n}(A) / \sim$ as the semigroup of equivalence classes of smooth cycles of degree $n$.

We will write $[\tilde{c}, \alpha]$ for the equivalence class of $(\tilde{c}, \alpha)$.

Lemma 4.8 $\widehat{M U}^{n}(A)$ is a group.

Proof Let $[\tilde{c}, \alpha] \in \widehat{M U}(A)$. It suffices to show that it admits an inverse. Since $M U(A)$ is a group there exists a cycle $c^{\prime}$ such that $c+c^{\prime} \sim 0$. By Lemma 3.20 we can assume that $c(l)+c^{\prime}(l) \sim \partial b$ for some bordism datum $b$ and $l \in \mathbb{N}$. We extend $b$ to a geometric bordism datum $\widetilde{b}$ by choosing a connection such that $\partial \tilde{b} \cong \widetilde{c}(l)+\widetilde{c}^{\prime}(l)$ for some geometric extension $\widetilde{c}^{\prime}$ of $c^{\prime}$. Then we have $\left[\widetilde{c}^{\prime}, T(\tilde{b})-\alpha\right]+[\widetilde{c}, \alpha]=0$. 
4.2.4 We now define the structure maps $a, R, I$ (see Definition 2.2) of the smooth extension $\widehat{M U}$.

Definition 4.9 (1) We define $R: \widehat{M U}(A) \rightarrow \Omega_{d=0}(A, R)$ by $R([\widetilde{c}, \alpha]):=T(\widetilde{c})-$ $d \alpha$.

(2) We define $a: \Omega(A, R) \rightarrow \widehat{M U}(A)$ by $a(\alpha):=[\varnothing,-\alpha]$.

(3) We define $I: \widehat{M U}(A) \rightarrow M U(A)$ by $I([\widetilde{c}, \alpha]):=[c]$ (using the geometric model Definition 3.19).

Lemma 4.10 These maps are well defined. We have $R \circ a=d$.

Proof The only nonobvious part is the fact that $R$ is well defined. To this end consider a geometric bordism datum $\widetilde{b}$. Then we have

$$
R[\partial \tilde{b}, T(\tilde{b})]=T(\partial \tilde{b})-d T(\tilde{b})=0
$$

by Equation (4-4).

4.2.5 We now extend $A \mapsto \widehat{M U}(A)$ to a contra-variant functor on the category of smooth manifolds. Let $f: B \rightarrow A$ be a smooth map. Then we must construct a functorial pullback $f^{*}: \widehat{M U}(A) \rightarrow \widehat{M U}(B)$ such that the transformations $R, I, a$ above become natural.

Let $(\tilde{c}, \alpha)$ be a smooth cycle with $\tilde{c}=(p, v), p: W \rightarrow A$. We can assume that $p$ is transverse to $f$. Otherwise we replace $p$ by a bordant (homotopic) map and correct $\alpha$ correspondingly so that the new pair represents the same class in $\widehat{M U}(A)$ as $(\tilde{c}, \alpha)$. Then we have the Cartesian diagram

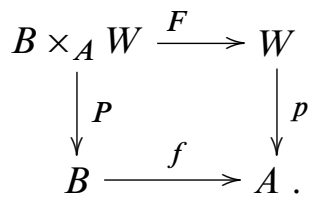

The map $P$ is the underlying map of a geometric cycle $f^{*} \tilde{c}=\left(P, f^{*} v\right)$, where $f^{*} v$ is the pullback of the geometric normal $G$-structure as defined in Definition 3.9. We want to define $f^{*}[\tilde{c}, \alpha]:=\left[f^{*} \tilde{c}, f^{*} \alpha\right]$. The problem is that $\alpha$ is a distribution. In order to define the pullback $f^{*} \alpha$ of a distributional form we need the additional assumption that $\mathrm{WF}(\alpha) \cap N(f)=\varnothing$, where $N(f) \subseteq T^{*} A \backslash 0_{A}$ is the normal set to $f$ given by

$$
N(f):=\operatorname{clo}\left\{\eta \in T^{*} A \backslash 0_{A} \exists b \in B \text { such that } f(b)=\pi(\eta) \text { and } d f(b)^{*} \eta=0\right\}
$$

(where $\pi: T^{*} A \rightarrow A$ is the projection), and $\operatorname{WF}(\alpha)$ denotes the wave front set of $\alpha$. The wave front set of a distributional form $\alpha$ on $A$ is a conical subset of $T^{*} A$ which 
measures the locus and the directions of the singularities of $\alpha$. For a precise definition and for the properties of distributions using the wave front set needed below we refer to Hörmander [15, Section 8]. Note that we can change $\alpha$ by exact forms with distribution coefficients without altering the class of $(\tilde{c}, \alpha)$. The idea is to show that one can choose $\alpha$ such that $\mathrm{WF}(\alpha) \cap N(f)=\varnothing$ holds. By [15, Theorem 8.2.4], in this case $f^{*} \alpha$ is defined. It is independent of the choice again up to exact forms with distribution coefficients. The details will be explained in the following lemmas.

Lemma 4.11 Let $\alpha \in \Omega_{-\infty}^{n}(A)$. Then there exists $\beta \in \Omega_{-\infty}^{n-1}(A)$ such that $\mathrm{WF}(\alpha-$ $d \beta) \subseteq \mathrm{WF}(d \alpha)$.

Proof We choose a Riemannian metric on $A$. Then we can define the formal adjoint $\delta:=d^{*}$ of the de Rham differential and the Laplacian $\Delta:=\delta d+d \delta$. Since $\Delta$ is elliptic we can choose a proper pseudo-differential parametrix $P$ of $\Delta$. This is a pseudodifferential operator of degree -2 which is an inverse of $\Delta$ up to pseudo-differential operators of degree $-\infty$ (smoothing operators). A pseudo-differential operator on $A$ is called proper if the restriction of the two projections from the support (a subset of $A \times A$ ) of its distribution kernel to the two factors $A$ are proper maps.

Then we form $G:=\delta P$. This pseudo-differential operator satisfies $d G+G d=1+S$, where $S$ is a proper smoothing operator. We thus can set $\beta:=G \alpha$ and have

$$
\alpha-d \beta=G d \alpha-S \alpha .
$$

Since $S \alpha$ is smooth and $\operatorname{WF}(G d \alpha) \subseteq \operatorname{WF}(d \alpha)$ (a pseudo-differential operator does not increase wave front sets) we see that $\mathrm{WF}(\alpha-d \beta) \subseteq \mathrm{WF}(d \alpha)$.

If $\alpha \in \Omega_{-\infty}(A, R)$, then we can write for some $s \in \mathbb{N}$

$$
\alpha=\sum_{i=1}^{s} \alpha_{i} \otimes r_{i}
$$

with $\alpha_{i} \in \Omega_{-\infty}^{n}$, and with linearly independent $r_{i} \in R$. In this case the wave front set of $\alpha$ is by definition $\operatorname{WF}(\alpha):=\bigcup_{i=1}^{s} \operatorname{WF}\left(\alpha_{i}\right)$. It is now easy to see that Lemma 4.11 extends to forms with coefficients in $R$.

Lemma 4.12 If $(\tilde{c}, \alpha), \tilde{c}=(p, v)$, is a smooth cycle, then we can choose $\alpha$ such that $\mathrm{WF}(\alpha) \subseteq N(p)$.

Proof It is a general fact that the wave front set of the pushforward of a smooth distribution along a map is contained in the normal set of the map. In view of Definition 
4.4 we have $\operatorname{WF}(T(\widetilde{c})) \subseteq N(p)$. Since $T(\widetilde{c})-d \alpha$ is smooth we have $\operatorname{WF}(d \alpha)=$ $\mathrm{WF}(T(\widetilde{c})) \subseteq N(p)$, and by Lemma 4.11 we can change $\alpha$ by an exact form such that $\mathrm{WF}(\alpha) \subseteq N(p)$.

4.2.6 A reformulation of the fact that $f$ and $p$ are transverse is that $N(f) \cap N(p)=\varnothing$. Using Lemma 4.12 we now take a representative of $\alpha$ such that $\operatorname{WF}(\alpha) \subseteq N(p)$. Then $f^{*} \alpha$ is a well defined distribution.

Definition 4.13 We define $f^{*}[\widetilde{c}, \alpha]=\left[f^{*} \tilde{c}, f^{*} \alpha\right]$, where we take representatives $\tilde{c}=(p, v)$ and $\alpha$ such that $f$ and $p$ are transverse and $\operatorname{WF}(\alpha) \subseteq N(p)$.

\section{Lemma 4.14 The pullback is well defined and functorial.}

Proof First we show that the pullback is well defined with respect to the choice of $\alpha$. Let $\beta \in \Omega_{-\infty}(A, R)$ and $\alpha^{\prime}:=\alpha+\beta$ be such that $T(\widetilde{c})-d \alpha^{\prime}$ is smooth. This implies that $\operatorname{WF}\left(\alpha^{\prime}\right) \subseteq N(p)$, and hence $\mathrm{WF}(d \beta) \subseteq N(p)$. By Lemma 4.11 we can modify $\beta$ by a closed form such that $\operatorname{WF}(\beta) \subseteq N(p)$. Then $f^{*} \alpha^{\prime}=f^{*} \alpha+d f^{*} \beta$.

It is easy to see that the pullback is additive and preserves stabilization. It remains to show that it preserves zero bordism. Let $\tilde{b}=((h, q), \mu)$ be a geometric bordism datum over $A$ with $(h, q): W \rightarrow \mathbb{R} \times A$. We define $W_{0}:=h^{-1}(\{0\})$ and assume that $q$ and $\left.q\right|_{W_{0}}$ are transverse to $f$. We then have the geometric bordism datum $\left(\operatorname{id}_{\mathbb{R}} \times f\right) * \widetilde{b}$ over $B$.

Let us define the normal datum of $b$ by

$$
\begin{aligned}
& N(b):=\operatorname{clo}\left\{\eta \in T^{*} A \backslash 0_{A} \exists v \in W\right. \text { such that } \\
& \left.\qquad E(v)=\pi(\eta) \text { and }\left(d E(v)^{*} \eta=0 \text { or } v \in W_{0} \text { and }\left.d E(v)^{*} \eta\right|_{T_{v} W_{0}}=0\right)\right\} .
\end{aligned}
$$

Then we have $\operatorname{WF}(T(\tilde{b})) \subseteq N(b)$. Again, since $q$ and $\left.q\right|_{W_{0}}$ are transverse to $f$ we have $N(b) \cap N(f)=\varnothing$ so that $f^{*} T(\tilde{b})$ is well defined. Using the fact that in a Cartesian diagram pushforward of distributions commutes with pullback we get $f^{*} T(\tilde{b})=T\left(f^{*} \tilde{b}\right)$. It follows that $\left(f^{*} \partial \tilde{b}, f^{*} T(\tilde{b})\right)=\left(\partial f^{*} \widetilde{b}, T\left(f^{*} \tilde{b}\right)\right)$. This implies that the pullback is well defined on the level of equivalence classes.

We now show functoriality. Let $g: C \rightarrow B$ be a second smooth map. If $\widehat{x} \in \widehat{M U}(A)$, then we can choose the representing smooth cycle $(\tilde{c}, \alpha)$ with $\tilde{c}=(p, v)$ such that $p$ is transverse to $f$ and $f \circ g$. In this case one easily sees that $(f \circ g)^{*}(\tilde{c}, \alpha)$ and $g^{*} f^{*}(\tilde{c}, \alpha)$ are isomorphic cycles. 
4.2.7 We now have defined a functor $A \mapsto \widehat{M U}(A)$ from smooth manifolds to graded groups.

Lemma 4.15 The transformations $R, I$ and $a$ are natural.

Proof Straightforward.

4.2.8 We now define the outer product

$$
\times: \widehat{M U}(A) \otimes \widehat{M U}(B) \rightarrow \widehat{M U}(A \times B) .
$$

Let $\hat{x} \in \widehat{M U}(A)$ be represented by $(\widetilde{c}, \alpha)$, and let $\hat{y} \in \widehat{M U}(B)$ be represented by $(\tilde{e}, \beta)$. In Definition 3.16 we have already defined the product of cycles $c \times e$. Here we enhance this definition to the geometric level. Write $\tilde{c}=(p, v)$ and $\tilde{d}=(q, \mu)$. Then we define $\tilde{c} \times \tilde{d}:=(p \times q, v \oplus \mu)$, where the sum of geometric normal $G$ structures $\nu \oplus \mu$ is defined similarly as in the nongeometric case.

Note that we have a graded outer product

$$
\times: \Omega_{-\infty}(A, R) \otimes \Omega_{-\infty}(B, R) \rightarrow \Omega_{-\infty}(A \times B, R) .
$$

Definition 4.16 We define the product of smooth cycles $(\tilde{c}, \alpha) \times(\widetilde{e}, \beta)$ by

$$
\left(\tilde{c} \times \tilde{e},(-1)^{|\hat{x}|} R(\hat{x}) \times \beta+\alpha \times T(e)\right),
$$

and we define the product $\hat{x} \times \hat{y} \in \widehat{M U}(A \times B)$ to be the corresponding equivalence class.

This cycle level definition needs a few verifications.

Lemma 4.17 (1) The outer product is well defined.

(2) It is associative, ie $(\hat{x} \times \hat{y}) \times \hat{z}=\hat{x} \times(\hat{y} \times \hat{z})$, where $\widehat{z} \in \widehat{M U}(C)$.

(3) It is graded commutative in the sense that $F^{*}(\hat{x} \times \hat{y})=(-1)^{|\hat{x}||\hat{y}|} \hat{y} \times \hat{x}$, where $F: B \times A \rightarrow A \times B$ is the flip $F(b, a):=(a, b)$.

(4) The product is natural, ie if $f: C \rightarrow A$ is a smooth map, then we have $f^{*} \hat{x} \times \hat{y}=$ $\left(f \times \operatorname{id}_{B}\right)^{*}(\hat{x} \times \hat{y})$.

Proof We first show that the cycle level definition of the outer product passes through the equivalence relation. It is obvious that the outer product is bilinear and preserves stabilizations in both arguments. It remains to verify that it preserves zero bordisms. 
Let $\tilde{b}$ be a geometric bordism datum. Then we can form the geometric bordism datum $\widetilde{b} \times \widetilde{e}$ (see Definition 3.16). We have $T(\widetilde{b} \times \widetilde{e})=T(\tilde{b}) \times T(\widetilde{e})$ so that

$$
\begin{aligned}
(\partial \tilde{b}, T(\tilde{b})) \times(\widetilde{e}, \beta) & =(\partial \tilde{b} \times \tilde{e}, T(\tilde{b}) \times T(\tilde{e})) \\
& =(\partial(\tilde{b} \times \widetilde{e}), T(\tilde{b} \times \tilde{e})) \\
& \sim 0 .
\end{aligned}
$$

In order to see that the product also preserves zero bordism in the second entry we rewrite

$$
(-1)^{|\widehat{x}|} R(\widehat{x}) \times \beta+\alpha \times T(\widetilde{e}) \stackrel{\operatorname{im}(d)}{\equiv}(-1)^{|\widehat{x}|} T(\widetilde{c}) \times \beta+\alpha \times R(\hat{y})
$$

and apply the same argument as above. Associativity, graded commutativity, and naturality hold true on the level of smooth cycles. To see this, for commutativity we use again (4-5), and the proof of associativity is based on similar calculations.

4.2.9 As usual, the outer product determines a graded commutative ring structure by restriction to the diagonal.

Definition 4.18 We define the ring structure on $\widehat{M U}(A)$ by $\hat{x} \cup \hat{y}:=\Delta^{*}(\hat{x} \times \hat{y})$, where $\Delta: A \rightarrow A \times A$ is the diagonal.

The following assertions are consequences of Lemmas 4.14 and 4.17.

Corollary 4.19 $A \mapsto \widehat{M U}(A)$ is a contravariant functor from the category of manifolds to the category of graded commutative rings.

Lemma 4.20 The transformations $R$ and $I$ are multiplicative, and $a(\alpha) \cup \hat{x}=$ $a(\alpha \wedge R(\hat{x}))$.

Proof Straightforward calculation.

4.2.10 Recall that we have fixed in Theorem 3.22, Lemma 3.26, Section 3.4.7 a graded ring $R$ over $\mathbb{R}$ and a formal power series $\phi \in R \llbracket z \rrbracket^{0}$ which determines an $R$-valued $U$-genus $r_{\phi}$.

Theorem 4.21 The functor $\widehat{M U}$ together with the transformations $R, I, a$ is a multiplicative smooth extension of the pair $\left(M U, r_{\phi}\right)$. 
Proof We must verify the properties required in Definition 2.2 and Definition 2.3. Most of them have been shown above. We are left with the commutativity of

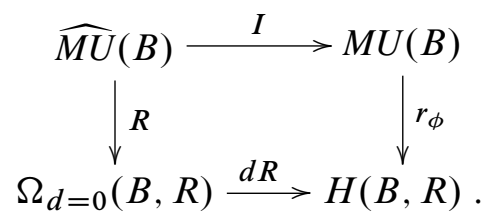

and the exactness of

$$
M U(B) \stackrel{r_{\phi}}{\rightarrow} \Omega(B, R) / \operatorname{im}(d) \stackrel{a}{\rightarrow} \widehat{M U}(B) \stackrel{I}{\rightarrow} M U(B) \rightarrow 0
$$

The commutativity of the diagram (4-6) is a direct consequence of (4-2).

We now discuss exactness of (4-7). We start with the surjectivity of $I$. Let $x \in M U(B)$ be represented by a cycle $c$. Then we can choose a geometric refinement $\tilde{c}$. We have $d T(\widetilde{c})=0$, and by Lemma 4.11 there exists $\alpha \in \Omega_{-\infty}(B, R)$ such that $T(\widetilde{c})-d \alpha$ is smooth. Therefore $(\tilde{c}, \alpha)$ is a smooth cycle, and we have $x=I[\tilde{c}, \alpha]$.

We now discuss exactness at $\widehat{M U}(B)$. It is clear that $I \circ a=0$. Let $\widehat{x} \in \widehat{M U}(B)$, be such that $I(\hat{x})=0$. Then we can assume that $\hat{x}$ is of the form $[\partial \tilde{b}, \alpha]$ for some geometric bordism datum $\tilde{b}$. Hence $\hat{x}=a(T(\tilde{b})-\alpha)$.

We now show exactness at $\Omega(B, R) / \operatorname{im}(d)$. Let $x \in M U(B)$ be represented by a cycle $c$. Then we choose a geometric refinement $\tilde{c}$, and by Lemma 4.11 a form $\alpha \in \Omega_{-\infty}(B, R)$ such that $T(\widetilde{c})-d \alpha$ is smooth. We have $r_{\phi}(x)=T(\widetilde{c})-d \alpha$. Let $c=(p, v)$ with $p: V \rightarrow B$, and consider the constant map $h: V \rightarrow \mathbb{R}$ with value 1 . The geometric normal $U$-structure of $(h, p): V \rightarrow \mathbb{R} \times B$ can also be represented by $\nu$. Then $\widetilde{b}=((h, p), v)$ is a geometric bordism datum with $\partial \widetilde{b}=\varnothing$ and $T(\widetilde{b})=T(\widetilde{c})$. It follows that

$$
a(d \alpha-T(\widetilde{c}))=[\partial \tilde{b}, T(\tilde{b})-d \alpha]=[\partial \tilde{b}, T(\tilde{b})]=0 .
$$

This proves that $a \circ r_{\phi}=0$.

Let now $\alpha \in \Omega(A, R)$ be such that $a(\alpha)=0$. Then there exist geometric bordism data $\widetilde{b}_{0}, \widetilde{b}_{1}$ such that $\partial \widetilde{b}_{0} \cong \partial \widetilde{b}_{1}$ and $T\left(\widetilde{b}_{0}\right)-T\left(\widetilde{b}_{1}\right)-\alpha \in \operatorname{im}(d)$. This already implies that $\alpha$ is closed. We construct a geometric cycle $\widetilde{c}$ such that $T(\widetilde{c})=T\left(\tilde{b}_{0}\right)-T\left(\tilde{b}_{1}\right)$ by gluing the bordism data along their common boundary. Then $[\alpha]=[T(\widetilde{c})]=r_{\phi}([c])$ in de Rham cohomology. 


\subsection{Smooth $M U$-orientations}

4.3.1 As before we fix a graded ring $R$ over $\mathbb{R}$ and a formal power series $\phi \in$ $R \llbracket z \rrbracket^{0}$. Let $\widehat{M U}$ be the smooth extension of $\left(M U, r_{\phi}\right)$ as in Theorem 4.21 with structure maps $R, a, I$. If $q: V \rightarrow A$ is a proper $M U$-oriented map, then we have an integration $q !: M U(V) \rightarrow M U(A)$ (see Section 3.3.10). Under the assumption that $q$ is a submersion we introduce the notion of a smooth $M U$-orientation and define the integration map $q !: \widehat{M U}(V) \rightarrow \widehat{M U}(A)$.

4.3.2 Let $q: V \rightarrow A$ be a proper submersion.

Definition 4.22 A representative of a smooth $M U$-orientation of $q$ is a pair $\check{c}:=(\tilde{c}, \sigma)$, where $\widetilde{c}$ is a geometric cycle with underlying map $q: V \rightarrow A$ and $\sigma \in \Omega^{-1}(V, R)$.

A representative of a smooth $M U$-orientation of $q$ induces in particular an $M U-$ orientation of $q$.

4.3.3 We now introduce an equivalence relation $\sim$ called stable homotopy on the set of representatives of smooth $M U$-orientations of $q$.

Definition 4.23 We define the $l$-fold stabilization of $(\widetilde{c}, \sigma)$ by $(\widetilde{c}, \sigma)(l):=(\widetilde{c}(l), \sigma)$.

Let $h_{i}: A \rightarrow \mathbb{R} \times A$ denote the inclusions $h_{i}(a):=(i, a), i=0,1$. Consider a geometric cycle $\tilde{d}=(p, \mu)$ over $\mathbb{R} \times A$ with underlying map $p:=\mathrm{id}_{\mathbb{R}} \times q: \mathbb{R} \times V \rightarrow \mathbb{R} \times A$. It gives rise to a closed form $\phi\left(\nabla^{\mu}\right) \in \Omega^{0}(\mathbb{R} \times V, R)$. Let $\widetilde{c}_{i}:=h_{i}^{*} \widetilde{d}, \widetilde{c}_{i}=\left(q, v_{i}\right)$.

Definition 4.24 We call $\tilde{d}$ a homotopy between $\tilde{c}_{0}$ and $\tilde{c}_{1}$.

Definition 4.25 We define the transgression form

$$
\tilde{\phi}\left(\nabla^{v_{1}}, \nabla^{v_{0}}\right):=\int_{[0,1] \times V / V} \phi\left(\nabla^{\mu}\right) \in \Omega^{-1}(A, R) / \operatorname{im}(d) .
$$

Since the underlying cycle $d$ of $\tilde{d}$ is a product, and since the space of geometric refinements of $d$ is contractible, the transgression form is well defined independent of the choice of the homotopy (this is a standard argument in the theory of characteristic forms). By Stokes' theorem the transgression satisfies

$$
d \tilde{\phi}\left(\nabla^{v_{1}}, \nabla^{v_{0}}\right)=\phi\left(\nabla^{v_{1}}\right)-\phi\left(\nabla^{v_{0}}\right) .
$$

Definition 4.26 We call two representatives of a smooth $M U$-orientation $\left(\widetilde{c}_{i}, \sigma_{i}\right)$ homotopic if there exists a homotopy $\tilde{d}$ from $\tilde{c}_{0}$ to $\tilde{c}_{1}$, and $\sigma_{1}-\sigma_{0}=\tilde{\phi}\left(\nabla^{v_{1}}, \nabla^{v_{0}}\right)$. 
4.3.4 We now define equivalence of representatives of smooth $M U$-orientations.

Definition 4.27 Let $\sim$ be the minimal equivalence relation on the set of representatives of smooth $M U$-orientations on $q$ such that

(1) $(\tilde{c}, \sigma) \sim(\widetilde{c}(l), \sigma)$

(2) $\left(\widetilde{c}_{0}, \sigma_{0}\right) \sim\left(\widetilde{c}_{1}, \sigma_{1}\right)$, if $\left(\widetilde{c}_{0}, \sigma_{0}\right)$ and $\left(\widetilde{c}_{1}, \sigma_{1}\right)$ are homotopic.

A smooth $M U$-orientation of $q$ is an equivalence class of representatives of smooth $M U$-orientations which we will usually write as $o:=[\tilde{c}, \sigma]$.

4.3.5 Let $\tilde{c}:=(q, v)$ and $\check{c}:=(\widetilde{c}, \sigma)$ be a representative of a smooth $M U$-orientation.

Definition 4.28 We define $A(\check{c}):=\phi\left(\nabla^{v}\right)-d \sigma \in \Omega^{0}(V, R)$.

Lemma 4.29 The form $A(\check{c})$ only depends on the smooth $M U$-orientation [ $\check{c}]$ represented by $\check{c}$.

Proof This immediately follows from (4-8) and the definition of homotopy.

Below we will write $A(o):=A(\check{c})$, where $o:=[\check{c}]$.

4.3.6 In the following two paragraphs we define the operations of pullback and composition of smooth $M U$-orientations. We start with the pullback. Let $f: B \rightarrow A$ be a smooth map which is transverse to $q$. Then we have the Cartesian diagram

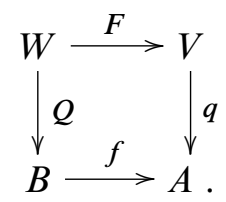

Definition 4.30 We define the pullback of a representative of a smooth $M U$-orientation of $q$ by $f^{*}(\tilde{c}, \sigma):=\left(f^{*} \tilde{c}, F^{*} \sigma\right)$ (see Section 4.2.5) which is a representative of a smooth $M U$-orientation of $Q$.

Lemma 4.31 The pullback is compatible with the equivalence relation. It induces a functorial pullback of smooth $M U$-orientations. We have $A\left(f^{*} o\right)=F^{*} A(o)$.

Proof It is clear that the pullback is compatible with stabilization. Let $\tilde{d}$ be a homotopy from $\tilde{c}_{0}$ to $\tilde{c}_{1}$. Then $\left(\operatorname{id}_{\mathbb{R}} \times f\right)^{*} \tilde{d}$ is a homotopy from $f^{*} \tilde{c}_{0}$ to $f^{*} \tilde{c}_{1}$. Furthermore, one checks that $\tilde{\phi}\left(\nabla^{f^{*} v_{1}}, \nabla f^{*} v_{0}\right)=f^{*} \tilde{\phi}\left(\nabla^{v_{1}}, \nabla^{v_{0}}\right)$. These formulas imply that the pullback preserves homotopic representatives of smooth $M U$-orientations. We conclude that the pullback is well defined on the level equivalence classes. Functoriality and the fact that $A\left(f^{*} o\right)=F^{*} A(o)$ are easy to see. 
4.3.7 We now define the composition of smooth $M U$-orientations. Let $p: A \rightarrow B$ be a second proper submersion, and let $(\tilde{d}, \theta), \widetilde{d}=(p, \mu)$, be a representative of a smooth $M U$-orientation of $p$. Let $o_{q}=[\tilde{c}, \sigma]$ and $o_{p}:=[\tilde{d}, \theta]$. By $\tilde{d} \circ \tilde{c}$ we denote the composition of geometric cycles which is based on Definition 3.11.

Definition 4.32 We define

$$
o_{p} \circ o_{q}:=\left[\tilde{d} \circ \tilde{c}, A\left(o_{q}\right) \wedge q^{*} \theta+\sigma \wedge q^{*} \phi\left(\nabla^{\mu}\right)\right] .
$$

The definition requires some verifications.

Lemma 4.33 The composition of smooth $M U$-orientations is well defined, compatible with pullback, and functorial.

Proof We first show that the composition is well defined. It is clear that the composition is compatible with stabilization. Let $\tilde{b}$ be a homotopy from $\tilde{c}_{0}$ to $\tilde{c}_{1}$. Then $\operatorname{pr}_{2}^{*} \tilde{d} \circ \tilde{b}$ is a homotopy from $\tilde{b} \circ \tilde{c}_{0}$ to $\tilde{b} \circ \tilde{c}_{1}$, where $\operatorname{pr}_{2}: \mathbb{R} \times B \rightarrow B$ is the projection. We further calculate (using the properties stated in Lemma 4.2)

$$
\begin{aligned}
\left(\sigma_{1}-\sigma_{0}\right) \wedge q^{*} \phi\left(\nabla^{\mu}\right) & =\tilde{\phi}\left(\nabla^{v_{1}}, \nabla^{v_{0}}\right) \wedge q^{*} \phi\left(\nabla^{\mu}\right) \\
& =\widetilde{\phi}\left(\nabla^{\mu \circ v_{1}}, \nabla^{\mu \circ v_{0}}\right) .
\end{aligned}
$$

This calculation implies that the composition $(\tilde{d}, \theta) \circ \ldots$ preserves homotopic representatives.

Let us now consider a homotopy $\widetilde{e}$ from $\tilde{d}_{0}$ to $\widetilde{d}_{1}$ We get a homotopy $\widetilde{e} \circ \tilde{c}$ from $\tilde{d}_{0} \circ \tilde{c}$ to $\tilde{d}_{1} \circ \tilde{c}$. Furthermore we rewrite (note that we work modulo $\operatorname{im}(d)$ )

$$
A\left(o_{q}\right) \wedge q^{*} \theta+\sigma \wedge q^{*} \phi\left(\nabla^{\mu}\right)=\phi\left(\nabla^{\nu}\right) \wedge q^{*} \theta+\sigma \wedge q^{*} A\left(o_{p}\right) .
$$

We have

$$
\begin{aligned}
\phi\left(\nabla^{v}\right) \wedge q^{*}\left(\theta_{1}-\theta_{0}\right) & =\phi\left(\nabla^{v}\right) \wedge q^{*} \widetilde{\phi}\left(\nabla^{\mu_{1}}, \nabla^{\mu_{0}}\right) \\
& =\tilde{\phi}\left(\nabla^{\mu_{1} \circ v}, \nabla^{\mu_{0} \circ v}\right) .
\end{aligned}
$$

Hence $\cdots \circ(\tilde{c}, v)$ preserves homotopic representatives. This finishes the proof that the composition is well defined.

4.3.8 The composition of smooth $M U$-orientations is associative and compatible with pullback. For completeness let us state the second fact in greater detail. Let $r: Q \rightarrow B$ be a map which is transverse to $q$ and $p \circ q$. Then we have the composition of pullback 
diagrams

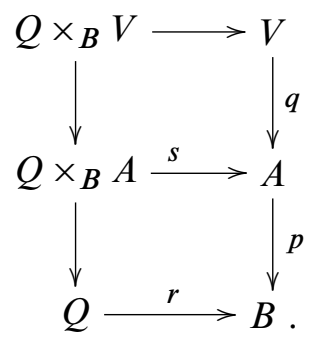

In this situation we have

$$
s^{*} o_{p} \circ r^{*} o_{q}=r^{*}\left(o_{p} \circ o_{q}\right) .
$$

We leave the details of the straightforward proof to the reader.

\subsection{The pushforward}

4.4.1 Let $p: V \rightarrow A$ be a proper submersion with a smooth $M U$-orientation $o_{p}:=$ $[\tilde{d}, \sigma], \tilde{d}=(p, v)$. In the following, $(\tilde{c}, \alpha)$ denotes a smooth cycle on $V$, and we use the notation

$$
\int_{V / A}:=p_{!}: \Omega_{-\infty}(V, R) \rightarrow \Omega_{-\infty}(A, R)
$$

for the integration of forms.

Definition 4.34 We define the pushforward on the level of cycles by

$$
p_{!}(\tilde{c}, \alpha)=\left(\tilde{d} \circ \tilde{c}, \int_{V / A}\left(\phi\left(\nabla^{v}\right) \wedge \alpha+\sigma \wedge R(\tilde{c}, \alpha)\right)\right) .
$$

Lemma 4.35 For fixed $(\tilde{d}, \sigma)$ the pushforward preserves equivalence of smooth cycles. Furthermore, the induced map $p_{!}: \widehat{M U}(V) \rightarrow \widehat{M U}(A)$ only depends on the equivalence class $[\tilde{d}, \sigma]$ of representatives of the smooth $M U$-orientation.

Proof It is clear that the pushforward is additive and compatible with stabilization. Let now $\widetilde{b}$ be a geometric bordism datum over $V$. Let pr: $\mathbb{R} \times A \rightarrow A$ be the projection and form $(\widetilde{e}, \theta):=\operatorname{pr}^{*}(\tilde{d}, \sigma)$. Then $\widetilde{e} \circ \tilde{b}$ is a bordism datum, and we have $T(\widetilde{e} \circ \tilde{b})=\int_{V / A} \phi\left(\nabla^{v}\right) \wedge T(\tilde{b})$. We calculate

$$
\begin{aligned}
p_{!}(\partial \tilde{b}, T(\tilde{b})) & =\left(\tilde{d} \circ \partial \tilde{b}, \int_{V / A} \phi\left(\nabla^{v}\right) \wedge T(\tilde{b})\right) \\
& =(\partial(\tilde{e} \circ \tilde{b}), T(\tilde{e} \circ \tilde{b})) .
\end{aligned}
$$

This equality implies that $p$ ! preserves zero bordisms. 
For a fixed representative $(\tilde{d}, \sigma)$ of the smooth $M U$-orientation we now have a well defined map $p !: \widehat{M U}(V) \rightarrow \widehat{M U}(A)$. Next we show that it only depends on the smooth orientation represented by $(\tilde{d}, \sigma)$. Again it is clear that stabilization of the representative of the smooth orientation does not change $p_{\text {! }}$. We now consider a homotopy $\widetilde{b}$ from $\left(\tilde{d}_{0}, \sigma_{0}\right)$ to $\left(\tilde{d}_{1}, \sigma_{1}\right)$. The idea of the argument is to translate this homotopy into a bordism datum. To this end we first consider a model case. Let $\kappa: \mathbb{R} \rightarrow \mathbb{R}$ be defined by $\kappa(x):=x-x^{2}$. Then $\kappa^{-1}(\{[0, \infty)\})=[0,1]$. We choose a representative of the stable normal bundle of $\kappa$ with a geometric $U$-structure $\mu$ such that $\widetilde{\kappa}=(\kappa, \mu)$ is a geometric bordism datum.

Let $\operatorname{pr}_{1}: \mathbb{R} \times A \rightarrow \mathbb{R}$ denote the projection. The composition $\tilde{r}:=\operatorname{pr}_{1}^{*} \tilde{\kappa} \circ \tilde{b}$ is now a bordism datum. Let $\rho$ denote the representative of the geometric $U$-structure on the normal bundle of $r$. We consider $\widetilde{r} \circ \mathrm{pr}_{2}^{*} \widetilde{c}$ as a geometric bordism datum with $\partial\left(\tilde{r} \circ \operatorname{pr}_{2}^{*} \widetilde{c}\right)=\tilde{d}_{0} \circ \tilde{c}+\left(\tilde{d}_{1} \circ \widetilde{c}\right)^{\mathrm{op}}$, where $(\cdot)^{\mathrm{op}}$ indicates a flip of the orientation. Fix $\tilde{c}=(q, v)$ with $q: U \rightarrow V$ and $\tilde{d}_{i}=\left(p, \lambda_{i}\right)$.

$$
\begin{aligned}
T\left(\tilde{r} \circ \operatorname{pr}_{2}^{*} \widetilde{c}\right) & =\int_{q^{-1} r^{-1}([0, \infty) \times V) / A} \phi\left(\nabla^{\rho}\right) \wedge \phi\left(\nabla^{v}\right) \\
& =\int_{V / A}\left(\tilde{\phi}\left(\nabla^{\lambda_{1}}, \nabla^{\lambda_{0}}\right) \wedge \int_{U / V} \phi\left(\nabla^{v}\right)\right)
\end{aligned}
$$

On the other hand

$$
\begin{aligned}
\int_{V / A}\left(\phi\left(\nabla^{\lambda_{1}}\right)-\phi\left(\nabla^{\lambda_{0}}\right)\right) \wedge \alpha & +\left(\sigma_{1}-\sigma_{0}\right) \wedge R(\tilde{c}, \alpha) \\
& =\int_{V / A} d \tilde{\phi}\left(\nabla^{\lambda_{1}}, \nabla^{\lambda_{0}}\right) \wedge \alpha+\tilde{\phi}\left(\nabla^{\lambda_{1}}, \nabla^{\lambda_{0}}\right) \wedge R(\tilde{c}, \alpha) \\
& =\int_{V / A} \tilde{\phi}\left(\nabla^{\lambda_{1}}, \nabla^{\lambda_{0}}\right) \wedge d \alpha+\tilde{\phi}\left(\nabla^{\lambda_{1}}, \nabla^{\lambda_{0}}\right) \wedge R(\tilde{c}, \alpha) \\
& =\int_{V / A}\left(\tilde{\phi}\left(\nabla^{\lambda_{1}}, \nabla^{\lambda_{0}}\right) \wedge \int_{U / V} \phi\left(\nabla^{v}\right)\right) .
\end{aligned}
$$

These two equations together show that $\left(\tilde{d}_{1}, \sigma_{1}\right) \circ(\tilde{c}, \alpha) \sim\left(d_{0}, \sigma_{0}\right) \circ(\tilde{c}, \alpha)$. Indeed

$$
\begin{aligned}
\left(\tilde{d}_{0} \circ \tilde{c}+\left(\tilde{d}_{1} \circ \tilde{c}\right)^{\mathrm{op}}, \int_{V / A}\left(\phi\left(\nabla^{\lambda_{1}}\right)-\phi\left(\nabla^{\lambda_{0}}\right)\right) \wedge \alpha\right. & \left.+\left(\sigma_{1}-\sigma_{0}\right) \wedge R(\tilde{c}, \alpha)\right) \\
& =\left(\partial\left(\tilde{r} \circ \operatorname{pr}_{2}^{*} \widetilde{c}\right), T\left(\widetilde{r} \circ \operatorname{pr}_{2}^{*} \widetilde{c}\right)\right) .
\end{aligned}
$$

4.4.2 We now establish the compatibility of the smooth pushforward with pushforward of forms and of bordism classes. 
Lemma 4.36 The following diagram commutes.

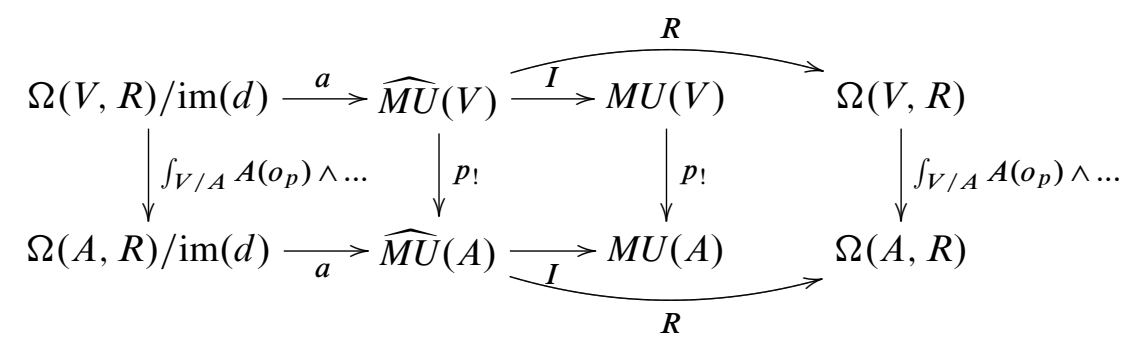

Proof Commutativity of the left square follows from partial integration

$$
\int_{V / A}\left(\phi\left(\nabla^{v}\right) \wedge \alpha-\sigma \wedge d \alpha\right)=\int_{V / A}\left(\phi\left(\nabla^{v}\right)-d \sigma\right) \wedge \alpha=\int_{V / A} A\left(o_{p}\right) \wedge \alpha .
$$

For the right square we use

$$
T(\tilde{d} \circ \widetilde{c})=\int_{V / A} \phi\left(\nabla^{v}\right) \wedge T(\widetilde{c})
$$

which implies

$$
\begin{aligned}
R\left(p_{!}(\tilde{c}, \alpha)\right) & =T(\tilde{d} \circ \tilde{c})-d \int_{V / A}\left(\phi\left(\nabla^{v}\right) \wedge \alpha+\sigma \wedge R(\tilde{c}, \alpha)\right) \\
& =\int_{V / A}\left(\phi\left(\nabla^{v}\right) \wedge T(\widetilde{c})-\phi\left(\nabla^{v}\right) \wedge d \alpha-d \sigma \wedge R(\tilde{c}, \alpha)\right) \\
& =\int_{V / A}\left(\phi\left(\nabla^{v}\right)-d \sigma\right) \wedge R(\tilde{c}, \alpha) \\
& =\int_{V / A} A\left(o_{p}\right) \wedge R(\tilde{c}, \alpha) .
\end{aligned}
$$

Commutativity of the middle square is a direct consequence of geometric description of $p_{!}: M U(V) \rightarrow M U(A)$ (see Section 3.3.10).

4.4.3 Let $p: V \rightarrow A$ be as before with the smooth $M U$-orientation $o_{p}:=[\tilde{d}, \sigma]$. We furthermore consider a proper submersion $q: A \rightarrow B$ with a smooth $M U$-orientation $o_{q}:=[\widetilde{e}, \rho], \tilde{e}=(q, \mu)$. Let $r:=q \circ p: V \rightarrow B$ be equipped with the composed smooth $M U$-orientation $o_{r}:=o_{q} \circ o_{p}$ (see Definition 4.32)

Lemma 4.37 The pushforward is functorial, ie we have the equality

$$
r_{!}=q_{!} \circ p_{!}: \widehat{M U}(V) \rightarrow \widehat{M U}(B) .
$$

Proof The equality holds on the smooth cycle level. The proof is a straightforward calculation of both sides by inserting the definitions and using the right square in Lemma 4.36 . 
4.4.4 Let $p: V \rightarrow A$ be a proper smoothly $M U$-oriented map as above, and let $f: B \rightarrow A$ be a second smooth map so that we get a Cartesian diagram

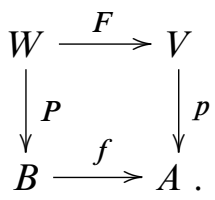

The map $P$ has an induced smooth $M U$-orientation $o_{P}:=f^{*} o_{p}$ (see Definition 4.30).

Lemma 4.38 The pushforward commutes with pullback, ie we have the equality

$$
P ! \circ F^{*}=f^{*} \circ p_{!}: \widehat{M U}(V) \rightarrow \widehat{M U}(B) .
$$

Proof The equality holds true on the level of smooth cycles $(\tilde{c}, \alpha)$ whose underlying map is transverse to $F$. By definition we have $o_{P}=\left[f^{*} \tilde{d}, f^{*} \sigma\right]$. Furthermore, it follows immediately from the definitions that $f^{*}(\tilde{d} \circ \widetilde{c})=f^{*} \tilde{d} \circ F^{*} \widetilde{c}$. The final ingredient of the verification is the identity

$$
f^{*} \circ \int_{V / A} \cdots=\int_{W / B} \circ F^{*} \cdots .
$$

4.4.5 Let $p: V \rightarrow A$ be a smoothly $M U$-oriented proper submersion as above.

Lemma 4.39 The projection formula holds true, ie for $x \in \widehat{M U}(A)$ and $y \in \widehat{M U}(V)$ we have $p_{!}\left(p^{*} x \cup y\right)=x \cup p ! y$.

Proof We consider the diagram

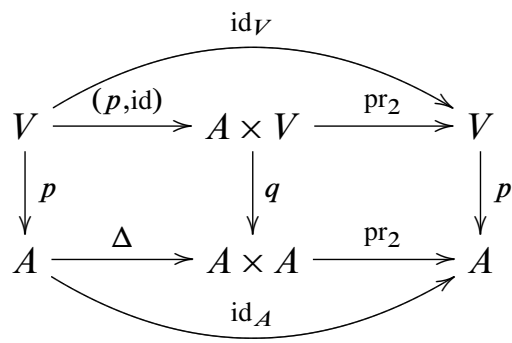

where $q:=\mathrm{id}_{A} \times p$ has the induced orientation $o_{q}:=\operatorname{pr}_{2}^{*} o_{p}$. If we show that

$$
q_{!}(x \times y)=x \times p_{!}(y),
$$

then by the definition of the cup-product and applying Lemma 4.38 to the left Cartesian square we get the result. Equation (4-9) holds true on the level of smooth cycles and is straightforward to check. 


\section{References}

[1] J F Adams, Stable homotopy and generalised homology, Chicago Lectures in Math., Univ. of Chicago Press (1974) MR0402720

[2] J-L Brylinski, Loop spaces, characteristic classes and geometric quantization, Progress in Math. 107, Birkhäuser, Boston (1993) MR1197353

[3] U Bunke, M Kreck, T Schick, A geometric description of smooth cohomology, to appear in Ann. Math. Blaise Pascal arXiv:0903.5290

[4] U Bunke, T Schick, Smooth $K$-theory, to appear in "From probability to geometry", (X Ma, editor) Asterisque, Volume dedicated to J-M Bismut for his 60-th birthday arXiv:0707.0046

[5] U Bunke, T Schick, Uniqueness of the extensions of generalized cohomology theories, submitted arXiv:0901.4423

[6] U Bunke, T Schick, On the topology of T-duality, Rev. Math. Phys. 17 (2005) 77-112 MR2130624

[7] J Cheeger, J Simons, Differential characters and geometric invariants, from: "Geometry and topology (College Park, Md., 1983/84)", (J Alexander, J Harer, editors), Lecture Notes in Math. 1167, Springer, Berlin (1985) 50-80 MR827262

[8] J L Dupont, R Ljungmann, Integration of simplicial forms and Deligne cohomology, Math. Scand. 97 (2005) 11-39 MR2179587

[9] J Franke, On the construction of elliptic cohomology, Math. Nachr. 158 (1992) 43-65 MR1235295

[10] D S Freed, Dirac charge quantization and generalized differential cohomology, from: "Surveys in differential geometry", (S-T Yau, editor), Surv. Differ. Geom. VII, Int. Press, Somerville, MA (2000) 129-194 MR1919425

[11] DS Freed, M Hopkins, On Ramond-Ramond fields and K-theory, J. High Energy Phys. (2000) Paper 44, 14 MR1769477

[12] P Gajer, Geometry of Deligne cohomology, Invent. Math. 127 (1997) 155-207 MR1423029

[13] F Hirzebruch, T Berger, R Jung, Manifolds and modular forms, Aspects of Math. E20, Friedr. Vieweg \& Sohn, Braunschweig (1992) MR1189136 With appendices by N-P Skoruppa and by P Baum

[14] M J Hopkins, I M Singer, Quadratic functions in geometry, topology, and M-theory, J. Differential Geom. 70 (2005) 329-452 MR2192936

[15] L Hörmander, The analysis of linear partial differential operators. I. Distribution theory and Fourier analysis, Classics in Math., Springer, Berlin (2003) MR1996773 Reprint of the second (1990) edition 
[16] P S Landweber, Homological properties of comodules over $\mathrm{MU}_{*}(\mathrm{MU})$ and $\mathrm{BP}_{*}(\mathrm{BP})$, Amer. J. Math. 98 (1976) 591-610 MR0423332

[17] P S Landweber, D C Ravenel, R E Stong, Periodic cohomology theories defined by elliptic curves, from: "The Čech centennial (Boston, MA, 1993)", (M Cenkl, H Miller, editors), Contemp. Math. 181, Amer. Math. Soc. (1995) 317-337 MR1320998

[18] J W Milnor, J D Stasheff, Characteristic classes, Annals of Math. Studies 76, Princeton Univ. Press (1974) MR0440554

[19] D Quillen, On the formal group laws of unoriented and complex cobordism theory, Bull. Amer. Math. Soc. 75 (1969) 1293-1298 MR0253350

[20] G de Rham, Differentiable manifolds. Forms, currents, harmonic forms, Grund. der Math. Wissenschaften 266, Springer, Berlin (1984) MR760450 Translated from the French by F R Smith, With an introduction by S S Chern

[21] J Simons, D Sullivan, Axiomatic characterization of ordinary differential cohomology, J. Topol. 1 (2008) 45-56 MR2365651

[22] R E Stong, Notes on cobordism theory, Math. notes, Princeton Univ. Press (1968) MR0248858

[23] R M Switzer, Algebraic topology—homotopy and homology, Classics in Math., Springer, Berlin (2002) MR1886843 Reprint of the 1975 original

UB: NWF I - Mathematik, Universität Regensburg 93040 Regensburg, Germany

TS, IS, MW: Mathematisches Institut, Georg-August-Universität Göttingen Bunsenstr 3, 37073 Göttingen, Germany

ulrich.bunke@mathematik.uni-regensburg.de, schick@uni-math.gwdg.de, ischroed@uni-math.gwdg.de

http://www . mathematik. uni-regensburg.de/Bunke/, http://www. uni-math.gwdg.de/schick

Received: 24 September 2008 Revised: 15 July 2009 